\title{
Values of Brownian intersection exponents, II: Plane exponents
}

\author{
by \\ GREGORY F. LAWLER \\ ODED SCHRAMM \\ and \\ WENDELIN WERNER \\ Duke University \\ Durham, NC, U.S.A. \\ Microsoft Research \\ Redmond, WA, U.S.A. \\ Université Paris-Sud \\ Orsay, France \\ and \\ The Weizmann Institute of Science \\ Rehovot, Israel
}

\section{Introduction}

This paper is the follow-up of the paper [27], in which we derived the exact value of intersection exponents between Brownian motions in a half-plane. In the present paper, we will derive the value of intersection exponents between planar Brownian motions (or simple random walks) in the whole plane.

This problem is very closely related to the more general question of the existence and value of critical exponents for a wide class of two-dimensional systems from statistical physics, including percolation, self-avoiding walks and other random processes. Theoretical physics predicts that these systems behave in a conformally invariant way in the scaling limit, and uses this fact to predict certain critical exponents associated to these systems. We refer to [27] for a more detailed account on this link and for more references on this subject.

Let us now briefly describe some of the results that we shall derive in the present paper. Suppose that $B^{1}, \ldots, B^{n}$ are $n \geqslant 2$ independent planar Brownian motions started from $n$ different points in the plane. Then it is easy to see (using a subadditivity argument) that there exists a constant $\zeta_{n}$ such that

$$
\mathbf{P}\left[\forall i \neq j \in\{1, \ldots, n\}, B^{i}[0, t] \cap B^{j}[0, t]=\varnothing\right]=t^{-\zeta_{n}+o(1)}
$$

when $t \rightarrow \infty$. We shall prove

The first author was supported by the National Science Foundation.

I. Benjamini, O. Häggström (eds.), Selected Works of Oded Schramm, Selected Works in Probability and Statistics, DOI 10.1007/978-1-4419-9675-6_29, C) Springer Science+Business Media, LLC 2011 
THEOREM 1.1. For all $n \geqslant 2$,

$$
\zeta_{n}=\frac{1}{24}\left(4 n^{2}-1\right)
$$

This result had been conjectured by Duplantier-Kwon [14] (see also, more recently, Duplantier [11]), using ideas from theoretical physics (conformal field theory, quantum gravity and analogies with some other models for which exponents had also been conjectured).

It was shown in [5] that the exponent $\zeta_{2}$ equals the corresponding exponent for simple random walks (see also [10]). This result was sharpened in [21], [26], where estimates were derived up to multiplicative constants. It follows from these results and Theorem 1.1 that if $S$ and $S^{\prime}$ denote two independent simple random walks started from neighboring vertices in $\mathbf{Z}^{2}$, then for some constant $c>0$,

$$
c^{-1} k^{-5 / 8} \leqslant \mathbf{P}\left[S[0, k] \cap S^{\prime}[0, k]=\varnothing\right] \leqslant c k^{-5 / 8}
$$

for all $k \geqslant 1$. Similarly, [20] and Theorem 1.1 imply

$$
c^{-1} t^{-5 / 8} \leqslant \mathbf{P}\left[B^{1}[0, t] \cap B^{2}[0, t]=\varnothing\right] \leqslant c t^{-5 / 8}
$$

for all $t \geqslant 1$, assuming that the distance between $B^{1}(0)$ and $B^{2}(0)$ is 1 , say.

One can define more general exponents, allowing intersection between some Brownian motions, but forbidding intersection between different packets of Brownian motions. For instance, there exists a constant $\zeta=\zeta(n, m)$ such that if $B^{1}, \ldots, B^{n}$ and $B^{\prime 1}, \ldots, B^{\prime m}$ denote $n+m$ independent planar Brownian motions started from points such that $B^{i}(0) \neq$ $B^{\prime j}(0)$ for all $i \leqslant n$ and $j \leqslant m$, then

$$
\mathbf{P}\left[\forall i \leqslant n, \forall j \leqslant m, B^{i}[0, t] \cap B^{\prime j}[0, t]=\varnothing\right]=t^{-\zeta+o(1)}
$$

when $t \rightarrow \infty$. Similarly, one can define exponents $\zeta\left(n_{1}, \ldots, n_{k}\right)$ corresponding to nonintersection between $k$ packets of Brownian motions.

It is easy to see (e.g. [23]) that there is a natural extension of the definition of $\zeta(n, m)$ to pairs $(n, \lambda)$, where $n$ is a positive integer and $\lambda$ is any positive real. In [31], it is shown that there is also a natural definition of $\zeta\left(\lambda_{1}, \ldots, \lambda_{k}\right)$ where the $\lambda_{j}$ are positive reals with $\lambda_{1}, \lambda_{2} \geqslant 1$. In the present paper, we shall derive the value of the exponents for a certain class of $k$-tuples $\left(\lambda_{1}, \ldots, \lambda_{k}\right)$ (see Theorem 5.3). In particular, we shall prove

THEOREM 1.2. For all real $\lambda \geqslant 2$,

$$
\zeta(2, \lambda)=\frac{1}{96}\left((5+\sqrt{24 \lambda+1})^{2}-4\right) .
$$


It has been shown by Lawler [20], [22], [23], [25] that some of these critical exponents are closely related to the Hausdorff dimension of exceptional subsets of a planar Brownian path. Recall that a cut point of a connected set $K$ is the set of points $x \in K$ such that $K \backslash\{x\}$ is disconnected. The Hausdorff dimension of the set of cut points of the Brownian path $B[0,1]$ is $2-2 \zeta_{2}$ almost surely [20]. Consequently, we get the following corollary from Theorem 1.1 .

Corollary 1.3. Let $B$ be Brownian motion in the plane. Then the Hausdorff dimension of the set of cut points of $B[0,1]$ is $\frac{3}{4}$ almost surely.

Recall that the frontier of a bounded set $K \subset \mathbf{R}^{2}$ is its outer boundary, i.e., the boundary of the unbounded connected component of $\mathbf{R}^{2} \backslash K$. The exponents $\zeta(2, \lambda)$ are closely related to the multifractal spectrum of the Brownian frontier [23]. In particular [22], the Hausdorff dimension $d_{F}$ of the frontier of $B[0,1]$ is almost surely $d_{F}=2-\eta_{2}$, where $\eta_{2}:=\lim _{\lambda \searrow 0} 2 \zeta(2, \lambda)$ is called the disconnection exponent for two Brownian paths. (This is not the definition of the disconnection exponent used in [22]; however, the two definitions are equivalent; see [24], [30].) It had been conjectured by Mandelbrot [35] (by analogy with the conjectures for planar self-avoiding walks) that $d_{F}=\frac{4}{3}$. Upper and lower bounds for $\eta_{2}$ from [6], [40], combined with the fact that $d_{F}=2-\eta_{2}$ [22], showed that $1.015<d_{F}<1.5$. (See also [4] for another proof of $d_{F}>1$.) In the present paper, we derive the values of $\zeta(2, \lambda)$ only for $\lambda \geqslant 2$, so that we cannot directly apply our results to show that $\eta_{2}=\frac{2}{3}$. However, in the subsequent paper [29], we prove

THEOREM 1.4. The function $\lambda \mapsto \zeta(2, \lambda)$ is real analytic on $(0, \infty)$.

Combining this with Theorem 1.2 shows immediately that (1.3) holds for all $\lambda>0$, and therefore $\eta_{2}=\frac{2}{3}$. This completes the proof of Mandelbrot's conjecture:

COROLlARY 1.5. The Hausdorff dimension of the Brownian frontier is almost surely $\frac{4}{3}$.

The formula for the multifractal spectrum of the Brownian frontier also follows. This formula has been conjectured in [31] as a consequence of the conjectures of DuplantierKwon [14] and of the functional relations between generalized Brownian exponents derived in [31]; see also recent physics work on this subject by Duplantier [11], [12].

The pioneer points of $B$ are the image under $B$ of the set of times $t$ such that $B(t)$ is in the frontier of $B[0, t]$. It has been shown [25] that the dimension of the set of pioneer points of $B$ is $2-\eta_{1}$, where $\eta_{1}:=\lim _{\lambda \searrow_{0}} 2 \zeta(1, \lambda)$. Below, we show that

$$
\zeta(1, \lambda)=\frac{1}{96}\left((3+\sqrt{24 \lambda+1})^{2}-4\right)
$$


for all sufficiently large $\lambda$ (see (5.10)). In [29] it will be proved that $\zeta(1, \lambda)$ is analytic for $\lambda>0$. Consequently, by analytic continuation of the above formula for $\zeta(1, \lambda)$, it follows that $\eta_{1}=\frac{1}{4}$. Hence, using the above-mentioned result of [25], we obtain

COROLLARY 1.6. The Hausdorff dimension of the set of pioneer points of Brownian motion is $\frac{7}{4}$ almost surely.

Let us briefly mention that there is a (non-rigorous) link between our results and the conjectures concerning two-dimensional self-avoiding walks. For instance, there is a heuristic argument (see [32]) which uses the Brownian intersection exponents and explains why the number of self-avoiding walks of length $N$ on a planar lattice increases asymptotically like $N^{11 / 32} \mu^{N}$, for some (lattice-dependent) constant $\mu>1$, as conjectured by Nienhuis [36] (see also [34] for a mathematical account).

Just as in [27], a central role in the present paper will be played by $\mathrm{SLE}_{6}$, the stochastic Löwner evolution process with parameter 6, which is conjectured [39] to correspond to the scaling limit of two-dimensional critical percolation cluster boundaries. In [39] the processes $\mathrm{SLE}_{\kappa}$ were introduced, and it was shown that $\mathrm{SLE}_{2}$ is the scaling limit of loop-erased random walk, assuming the conjecture that the latter has a conformally invariant scaling limit.

Actually, there are two versions of $\mathrm{SLE}_{\kappa}$. In the first version, which we now call radial $\mathrm{SLE}_{\kappa}$, one has a set $K_{t}$ growing from a boundary point of the unit disk to the interior point 0 , while in chordal $\mathrm{SLE}_{\kappa}$, the set $K_{t}$ grows from a point in $\mathbf{R}$ to $\infty$ within the upper half-plane. (The precise definitions will be recalled in §2.2.) By applying conformal maps, these processes can be defined in any simply-connected proper subdomain of the plane.

After recalling the definition of $\mathrm{SLE}_{\kappa}$, we study in $\S 3$ some of its properties. In particular, the $\mathrm{SLE}_{\kappa}$-analogues of the exponents $\zeta(1, \lambda), \lambda \geqslant 1$, are computed. From Cardy's formula for $\mathrm{SLE}_{6}$ (that we proved in [27]) we then derive the asymptotic decay of the probability that $\mathrm{SLE}_{6}$ crosses a long rectangle without touching the upper and lower boundaries of this rectangle, and show that chordal and radial $\mathrm{SLE}_{6}$ are very closely related.

We then turn our attention to the Brownian intersection exponents. In $\S 5$, the definition and some properties of the exponents are recalled. In particular, it is explained how to formulate these exponents in terms of non-intersection between two-dimensional Brownian excursions. Then, these facts (properties of $\mathrm{SLE}_{6}$, exponents for $\mathrm{SLE}_{6}$, description of the Brownian exponents in terms of Brownian excursions, properties of these Brownian excursions) are combined to derive the value of the Brownian intersection exponents. 


\section{Preliminaries}

\subsection{Notation}

Throughout this paper, $\mathbf{U}$ will denote the unit disk in the complex plane $\mathbf{C} . C=C_{1}=\partial \mathbf{U}$ will denote the unit circle. For any $r>0, C_{r}=r C_{1}$ will denote the circle of radius $r$ centered at 0 . $\mathbf{H}$ will denote the upper half-plane $\mathbf{H}=\{x+i y: y>0\}$. When $w \neq w^{\prime}$ are two points on the unit circle $C$, then $a\left(w, w^{\prime}\right)$ will denote the counterclockwise arc of $C$ from $w$ to $w^{\prime}$.

Just as in [31], [32], [27], it will be convenient to use $\pi$-extremal distances of quadrilaterals: this just means $\pi$ times the usual extremal distance. (Extremal distance is also known as extremal length. See, e.g., [1] for the definition and basic properties of extremal length and conformal maps.) The $\pi$-extremal distance between sets $A$ and $B$ in a set $O$ will be denoted by $\ell(A, B, O)$.

Let $f$ and $g$ be functions, and let $l \in \mathbf{R}$ or $l=\infty$. We say that $f(x) \sim g(x)$ when $x \rightarrow l$, if $f(x) / g(x) \rightarrow 1$. We write $f(x) \approx g(x)$, if $\log f(x) / \log g(x) \rightarrow 1$, and we write $f(x) \asymp g(x)$, if $f(x) / g(x)$ is bounded above and below by positive finite constants when $x$ is sufficiently close to $l$.

\subsection{Radial and chordal SLE}

In [27], we studied chordal $\mathrm{SLE}_{\kappa}$ as a random increasing family $\left(K_{t}, t \geqslant 0\right)$ of bounded subsets of the upper half-plane $\mathbf{H}$ (or, more generally, their image under a conformal map). As $t$ increases, the set $K_{t}$ grows, and $\bigcup_{t} K_{t}$ is unbounded. One can say that $K_{t}$ is growing towards $\infty$, which we think of as a boundary point of $\mathbf{H}$.

Similarly, when looking at the conformal image of $\left(K_{t}, t \geqslant 0\right)$ under the map $\varphi(z)=$ $(z-i) /(z+i)$ that maps $\mathbf{H}$ onto the unit disk and $\infty$ to 1 , we get an increasing family of subsets of the unit disk that is growing towards 1 .

In the present paper, we will mainly use a variant of this process, called radial $\mathrm{SLE}_{\kappa}$, where $\left(K_{t}, t \geqslant 0\right)$ is an increasing family of subsets of the unit disk that grows towards 0 . The main distinction is that 0 is an interior point of $\mathbf{U}$, instead of a boundary point.

Suppose that $\left(\delta_{t}, t \geqslant 0\right)$ is a continuous function taking values on the unit circle $C_{1}=\partial \mathbf{U}$. Consider for each $z \in \mathbf{U}$ the solution $g_{t}=g_{t}(z)$ of the ordinary differential equation

$$
\partial_{t} g_{t}=g_{t} \frac{\delta_{t}+g_{t}}{\delta_{t}-g_{t}}, \quad t \geqslant 0,
$$

with $g_{0}=z$. This equation (and the corresponding equation for $g_{t}^{-1}$ ) was first considered by Löwner (see [33], also [37]) and is called Löwner's differential equation. For each $z \in \mathbf{U}$, it is well defined up to the time $\tau_{z}$ where $\lim _{t} \tau_{\tau_{z}} g_{t}=\delta_{\tau_{z}}$, if there is such a $\tau_{z}$, 
and otherwise $\tau_{z}=\infty$. Let $D_{t}$ be the set of $z \in \mathbf{U}$ such that $t<\tau_{z}$ (i.e., the set on which $g_{t}$ is defined), and $K_{t}=\mathbf{U} \backslash D_{t}$. It is easy to check that $g_{t}$ is the unique conformal map from $D_{t}$ onto $\mathrm{U}$ such that $g_{t}(0)=0$ and $g_{t}^{\prime}(0)$ is a positive real number. (The notation $g^{\prime}$ refers to differentiation with respect to $z$.) It is also easy to verify that $g_{t}^{\prime}(0)=\exp (t)$ by differentiating both sides of (2.1) with respect to $z$ at $z=0$ and noting that $g_{t}(0)=0$.

Let $\left(B_{t}, t \geqslant 0\right)$ be Brownian motion on the real line, starting at some point $B_{0} \in \mathbf{R}$, and let $\kappa \geqslant 0$. Set $\delta_{t}:=\exp \left(i \sqrt{\kappa} B_{t}\right)$ and consider the solution to Löwner's differential equation as defined above. (Note that $\delta_{t}$ is just Brownian motion on $\partial \mathbf{U}$ with time scaled by $\kappa$.) The resulting process was defined in [39] and called $\mathrm{SLE}_{\kappa}$ (this acronym stands for stochastic Löwner evolution with parameter $\kappa$ ). The set $K_{t}$ is called the hull of $\mathrm{SLE}_{\kappa}$, and $\left(\delta_{t}\right)_{t \geqslant 0}$ its driving process.

If $f: D \rightarrow \mathbf{U}$ is a conformal map, then radial $\mathrm{SLE}_{\kappa}$ in $D$ starting from $f$ can be defined as the composition $g_{t} \circ f$, where $g_{t}$ is radial $\mathrm{SLE}_{\kappa}$ in $\mathbf{U}$. Its hull is $f^{-1}\left(K_{t}\right)$, where $K_{t}$ is the hull of $g_{t}$. If $\partial D$ is sufficiently tame, then $f^{-1}$ extends continuously to $\partial \mathbf{U}$, and hence $f^{-1}\left(\delta_{0}\right)$ is well defined, where $\delta$ is the driving parameter of $g_{t}$. We may then refer to the resulting $\mathrm{SLE}_{\kappa}$-process in $D$ as $\mathrm{SLE}_{\kappa}$ from $f^{-1}\left(\delta_{0}\right)$ to $f^{-1}(0)$ in $D$.

In [39], another variant SLE was also defined (see also [27]), which we now call chordal SLE . . Let $\mathbf{H}$ be the upper half-plane, let $\delta_{t}=\sqrt{\kappa} B_{t}$, and consider for each $z \in \mathbf{H}$ the solution $\tilde{g}_{t}=\tilde{g}_{t}(z)$ of the differential equation

$$
\partial_{t} \tilde{g}_{t}=\frac{-2}{\delta_{t}-\tilde{g}_{t}}, \quad t \geqslant 0
$$

with $\tilde{g}_{0}(z)=z$. As before, let $\widetilde{D}_{t}$ be the set of points $z \in \mathbf{H}$ for which (2.2) has a solution in some interval $\left[0, t^{\prime}\right], t^{\prime}>t$, and let $\widetilde{K}_{t}:=\mathbf{H} \backslash \widetilde{D}_{t}$. Then the process $\left(\tilde{g}_{t}\right)_{t \geqslant 0}$ is chordal $\mathrm{SLE}_{\kappa}$ in $\mathbf{H}$, and $\widetilde{K}_{t}$ is its hull. Recall that $\widetilde{K}_{t}$ is bounded for each $t$, but $\bigcup_{t \geqslant 0} \widetilde{K}_{t}$ is unbounded. If $f: D \rightarrow \mathbf{U}$ is a conformal map, then $f \circ g_{t}$ is called chordal $\mathrm{SLE}_{\kappa}$ in $D$. Its hull is $f^{-1}\left(K_{t}\right)$, where $K_{t}$ is the hull of the process $g_{t}$. If $\partial D$ is sufficiently tame, $f^{-1}(\infty)$ and $f^{-1}\left(\delta_{0}\right)$ are well defined. In this case, we refer to the process $f \circ g_{t}$ as $\mathrm{SLE}_{\kappa}$ from $f^{-1}\left(\delta_{0}\right)$ to $f^{-1}(\infty)$ in $D$. This terminology is explained more fully in [27].

For the main results of this paper, only the case $\kappa=6$ will be used. As we shall see in $\S 4.1$, radial $\mathrm{SLE}_{6}$ is essentially the same process as chordal $\mathrm{SLE}_{6}$. For $\kappa \neq 6$, the radial and chordal $\mathrm{SLE}_{\kappa}$-processes are closely related, but the equivalence is weaker.

\section{Annulus-crossing exponents for SLE}

\subsection{Statement}

Consider a radial $\mathrm{SLE}_{\kappa}$-process (with $\kappa \geqslant 0$ ) in the unit disk $\mathbf{U}$, with driving element $\delta_{t}$ starting at $\delta_{0}=1$. As described before, let $g_{t}$ be the conformal map $g_{t}: D_{t} \rightarrow \mathbf{U}$, with 
$g_{t}(0)=0$ and $g_{t}^{\prime}(0)=\exp (t)$. Let

$$
A_{t}:=\partial \mathbf{U} \backslash \bar{K}_{t}
$$

It is easy to see that $A_{t}$ is either an arc on $\partial \mathbf{U}$, or $A_{t}=\varnothing$.

Let $b \geqslant 0$, and set

$$
\nu=\nu(\kappa, b):=\frac{1}{16}\left(8 b+\kappa-4+\sqrt{(\kappa-4)^{2}+16 b \kappa}\right) .
$$

THEOREM 3.1. Let $\kappa>0$ and $r \in(0,1)$, and let $T(r)$ be the least $t>0$ such that $K_{t}$ intersects the circle $C_{r}$. Let $\mathfrak{L}(r)$ be the $\pi$-extremal distance from $C_{r}$ to $C_{1}$ in $\mathbf{U} \backslash K_{T(r)}$ (we set $\mathfrak{L}(r):=\infty$ if $A_{t}=\varnothing$ ). Then for $b \geqslant 1$, as $r \rightarrow 0$,

$$
\mathbf{E}[\exp (-b \mathfrak{L}(r))] \asymp r^{\nu}
$$

This theorem gives an analogue of Theorem 3.1 in [27] for crossing an annulus. Its proof and usage will also be similar.

Remarks. The constants implicit in the $\asymp$-notation may depend on $\kappa$ and $b$.

One can also show that the theorem holds when $b \in(0,1)$, but we only need the case $b \geqslant 1$ (and $\kappa=6$ ) in the present paper. Observe that the case $\kappa=0$ is also correct, and easy to verify, for then $K_{T(r)}=[r, 1]$.

One should also note that the values of the exponents for $\kappa=2$ fit with the conjecture that $\mathrm{SLE}_{2}$ is the scaling limit of two-dimensional loop-erased random walks [39] and the exponents computed by Kenyon [16], [17], [18] for loop-erased walks. For instance, the definition of loop-erased random walks suggests that the number of vertices in the loop erasure of an $N^{2}$-step walk is roughly $N^{2-\nu(2,1)}=N^{5 / 4}$ (loosely speaking, in order for one of the $N^{2}$ steps of the simple random walk to remain in the loop erasure, the future of the random walk beyond that step has to avoid the past loop-erased walk), in accordance with Kenyon's results.

Similarly, combining this result (with $\kappa=6$ ) with the non-intersection exponents between $\mathrm{SLE}_{6}$ in a rectangle (see the discussion at the end of [27]) and the restriction property for $\mathrm{SLE}_{6}$ (which was proved for chordal $\mathrm{SLE}_{6}$ in [27] and will be proved for radial $\mathrm{SLE}_{6}$ in $\S 4$ ) leads to the value of non-intersection exponents between independent $\mathrm{SLE}_{6}$ in an annulus that agree with predictions for annulus crossings in a critical percolation cluster (see [15], [9], [2]).

The proof of Theorem 3.1 will consist of three steps. First, we will obtain an estimate on $\mathbf{E}\left[\left|g_{t}^{\prime}\left(e^{i x}\right)\right|^{b}\right]$ for large (deterministic) times. We deduce from it a result concerning the large-time behavior of the arc length of $g_{t}\left(A_{t}\right)$, and then show that this implies Theorem 3.1. 


\subsection{Derivative exponents}

Lemma 3.2. Assume that $\kappa>0$ and $b>0$. Let $\mathcal{H}(x, t)$ denote the event $\left\{\exp (i x) \in A_{t}\right\}$, and set

$$
\begin{aligned}
f(x, t) & :=\mathbf{E}\left[\left|g_{t}^{\prime}(\exp (i x))\right|^{b} 1_{\mathcal{H}(x, t)}\right] \\
q=q(\kappa, b) & :=\frac{\kappa-4+\sqrt{(\kappa-4)^{2}+16 b \kappa}}{2 \kappa}, \\
h^{*}(x, t) & :=\exp (-t \nu)\left(\sin \left(\frac{1}{2} x\right)\right)^{q}
\end{aligned}
$$

where $\nu$ is as in (3.1). Then there is a constant $c>0$ such that

$$
\forall t \geqslant 1, \forall x \in(0,2 \pi), \quad h^{*}(x, t) \leqslant f(x, t) \leqslant c h^{*}(x, t) .
$$

Proof. Let $\delta_{t}=\exp \left(i \sqrt{\kappa} B_{t}\right)$ be the driving process of the $\mathrm{SLE}_{\kappa}$, with $B_{0}=0$. For all $x \in(0,2 \pi)$, let $Y_{t}^{x}$ be the continuous real-valued function of $t$ which satisfies

$$
g_{t}\left(e^{i x}\right)=\delta_{t} \exp \left(i Y_{t}^{x}\right)
$$

and $Y_{0}^{x}=x$. The function $Y_{t}^{x}$ is defined on the set of pairs $(x, t)$ such that $\mathcal{H}(x, t)$ holds. Since $g_{t}$ satisfies Löwner's differential equation

$$
\partial_{t} g_{t}(z)=g_{t}(z) \frac{\delta_{t}+g_{t}(z)}{\delta_{t}-g_{t}(z)}
$$

we find that

$$
d Y_{t}^{x}=\sqrt{\kappa} d B_{t}+\cot \left(\frac{1}{2} Y_{t}^{x}\right) d t
$$

Let

$$
\tau^{x}:=\inf \left\{t \geqslant 0: Y_{t}^{x} \in\{0,2 \pi\}\right\}
$$

denote the time at which $\exp (i x)$ is absorbed by $\bar{K}_{t}$, and define for all $t<\tau^{x}$

$$
\Phi_{t}^{x}:=\left|g_{t}^{\prime}(\exp (i x))\right|
$$

On $t \geqslant \tau^{x}$ set $\Phi_{t}^{x}:=0$. Note that on $t<\tau^{x}$,

$$
\Phi_{t}^{x}=\partial_{x} Y_{t}^{x}
$$

By differentiating (3.2) with respect to $z$, we find that for $t<\tau^{x}$,

$$
\partial_{t} \log \Phi_{t}^{x}=-\frac{1}{2 \sin ^{2}\left(\frac{1}{2} Y_{t}^{x}\right)},
$$


and hence (since $\Phi_{0}^{x}=1$ ),

$$
\left(\Phi_{t}^{x}\right)^{b}=\exp \left(-\frac{1}{2} b \int_{0}^{t} \frac{d s}{\sin ^{2}\left(\frac{1}{2} Y_{s}^{x}\right)}\right)
$$

for $t<\tau^{x}$.

We now show that the right-hand side of (3.5) is 0 when $t=\tau^{x}$. For all $x \in(0,2 \pi)$, choose $m \in \mathbf{N}$ such that $x \in\left(2^{-m}, 2 \pi-2^{-m}\right)$, and define for all $n \geqslant m$ the stopping times

$$
\begin{aligned}
& \varrho_{n}:=\inf \left\{t>0: Y_{t}^{x} \notin\left(2^{-n}, 2 \pi-2^{-n}\right)\right\}, \\
& \varrho_{n}^{\prime}:=\inf \left\{t>\varrho_{n}:\left|Y_{t}^{x}-Y_{\varrho_{n}}^{x}\right| \geqslant 2^{-n-1}\right\},
\end{aligned}
$$

and the event $\mathcal{R}_{n}:=\left\{\varrho_{n}^{\prime}-\varrho_{n}>4^{-n}\right\}$. It is easy to see (for instance by comparing $Y^{x}$ with two Bessel processes and using their scaling property, or alternatively, by comparing with Brownian motions with constant drifts; see, e.g., [38] for the definition of Bessel processes) that there is some constant $c^{\prime}>0$ such that for every $n \geqslant m$,

$$
\mathbf{P}\left[\mathcal{R}_{n}\right]>c^{\prime}
$$

The strong Markov property shows that the events $\left(\mathcal{R}_{m}, \mathcal{R}_{m+1}, \ldots\right)$ are independent, so that almost surely there exist infinitely many values of $n \in \mathbf{N}$ such that $\mathcal{R}_{n}$ holds. For these values of $n$,

$$
\int_{\varrho_{n}}^{\varrho_{n+1}} \frac{d t}{\sin ^{2}\left(\frac{1}{2} Y_{t}^{x}\right)} \geqslant \frac{4^{-n}}{\sin ^{2}\left(2^{-n}\right)} \geqslant 1
$$

Hence, almost surely,

$$
\int_{0}^{\tau^{x}} \frac{d t}{\sin ^{2}\left(\frac{1}{2} Y_{t}^{x}\right)}=\infty
$$

A similar argument shows that

$$
\lim _{x \searrow 0} f(x, t)=\lim _{x \nearrow 2 \pi} f(x, t)=0
$$

holds for all fixed $t>0$. Suppose, for instance, that $x \leqslant 2^{-n_{0}} \min \left(\sqrt{t}, \frac{1}{2} \pi\right)$, define $\varrho_{0}^{\prime \prime}=0$, $x_{0}=x$, and for all $n \geqslant 1$,

$$
\varrho_{n}^{\prime \prime}=\inf \left\{s \geqslant \varrho_{n-1}^{\prime \prime}: s=\varrho_{n-1}^{\prime \prime}+\left(x_{n-1}\right)^{2} \text { or }\left|Y_{s}^{x}-x_{n-1}\right| \geqslant \frac{1}{2} x_{n-1}\right\}
$$

and $x_{n}=Y_{\varrho_{n}^{\prime \prime}}^{x}$. Clearly, for all $n \leqslant n_{0}, 0<x_{n}<\frac{1}{2} \pi$ and $\varrho_{n}^{\prime \prime}<t$. By comparing $Y^{x}$ with Bessel processes or Brownian motions with constant drift, it is easy to check that for some (sufficiently small) $c>0$ (independent of $n$ and $x$ ), if we define the events $\mathcal{R}_{n}^{\prime}:=$ 
$\left\{\varrho_{n}^{\prime \prime}=\varrho_{n-1}^{\prime \prime}+\left(x_{n-1}\right)^{2}\right\}$, then for all $n \leqslant n_{0}, \mathbf{P}\left[\mathcal{R}_{n}^{\prime} \mid \mathcal{F}_{n-1}\right] \geqslant c^{\prime}$, where $\mathcal{F}_{n-1}$ denotes the $\sigma$ field generated by the events $\mathcal{R}_{1}^{\prime}, \ldots, \mathcal{R}_{n-1}^{\prime}$. It therefore easily follows that when $x \searrow 0$,

$$
\int_{0}^{\min \left\{\tau^{x}: t\right\}} \frac{d s}{\sin ^{2}\left(\frac{1}{2} Y_{s}^{x}\right)} \rightarrow \infty
$$

in probability, and therefore also that $f(x, t) \rightarrow 0$.

Let $F:[0,2 \pi] \rightarrow \mathbf{R}$ be a continuous function with $F(0)=F(2 \pi)=0$, which is smooth in $(0,2 \pi)$, and set

$$
h(x, t)=h_{F}(x, t):=\mathbf{E}\left[\left(\Phi_{t}^{x}\right)^{b} F\left(Y_{t}^{x}\right)\right] .
$$

By (3.5) and the general theory of diffusion Markov processes (see, e.g., [3]), we know that $h$ is smooth in $(0,2 \pi) \times \mathbf{R}_{+}$. From the Markov property for $Y_{t}^{x}$ and (3.5), it follows that $h\left(Y_{t}^{x}, t^{\prime}-t\right)\left(\Phi_{t}^{x}\right)^{b}$ is a local martingale on $t<\min \left\{\tau^{x}, t^{\prime}\right\}$. Consequently, the drift term of the stochastic differential $d\left(h\left(Y_{t}^{x}, t^{\prime}-t\right)\left(\Phi_{t}^{x}\right)^{b}\right)$ is zero at $t=0$. By Itô's formula, this means

$$
\partial_{t} h=\Lambda h,
$$

where

$$
\Lambda h:=\frac{1}{2} \kappa \partial_{x}^{2} h+\cot \left(\frac{1}{2} x\right) \partial_{x} h-\frac{b}{2 \sin ^{2}\left(\frac{1}{2} x\right)} h .
$$

It can be verified directly that $h^{*}$ from the statement of the lemma solves (3.8). We therefore choose

$$
F(x):=\left(\sin \left(\frac{1}{2} x\right)\right)^{q}
$$

and claim that $h^{*}=h_{F}$. Indeed, both satisfy (3.8) on $[0,2 \pi] \times[0, \infty)$, and $h^{*}(x, 0)=F(x)=$ $h_{F}(x, 0)$ on $[0,2 \pi]$. Moreover, $F \leqslant 1$ implies that $h_{F} \leqslant f$ everywhere, so $h^{*}-h_{F}=0$ on $\{0,2 \pi\} \times(0, \infty)$, by $(3.7)$. It is also immediate to verify that $h_{F}(x, t) \rightarrow 0$ as $(x, t) \rightarrow(0,0)$ or $(x, t) \rightarrow(2 \pi, 0)$. Set $M=h_{F}-h^{*}$. Then $M$ is smooth in $(0,2 \pi) \times(0, \infty)$, continuous on $[0,2 \pi] \times[0, \infty)$, and satisfies $\partial_{t} M=\Lambda M$.

The proof that $M=0$ can be viewed as a straightforward application of the maximum principle. Fix some $\varepsilon>0$, and suppose that $M \geqslant \varepsilon$ at some point $(x, t) \in[0,2 \pi] \times[0, \infty)$. Among such points, let $\left(x_{0}, t_{0}\right)$ be a point with $t_{0}$ minimal. It is clear that there must be such a minimal point and that $x_{0} \in(0,2 \pi), t_{0}>0$. At $\left(x_{0}, t_{0}\right)$ we must have $\partial_{t} M \geqslant 0$, by minimality of $t_{0}$. Similarly, $\partial_{x} M\left(x_{0}, t_{0}\right)=0, \partial_{x}^{2} M\left(x_{0}, t_{0}\right) \leqslant 0$ and $M\left(x_{0}, t_{0}\right)=\varepsilon$. However, this gives $0 \leqslant \partial_{t} M\left(x_{0}, t_{0}\right)=(\Lambda M)\left(x_{0}, t_{0}\right) \leqslant-b \varepsilon / 2 \sin ^{2}\left(\frac{1}{2} x_{0}\right)$, by the definition of the operator $\Lambda$, a contradiction. Since $\varepsilon$ was arbitrary, this gives $M \leqslant 0$. The same argument applied to $-M$ shows that $M \geqslant 0$, which verifies (the subscript will henceforth be omitted from $h_{F}$ )

$$
h(x, t)=h^{*}(x, t)=\exp (-t \nu)\left(\sin \left(\frac{1}{2} x\right)\right)^{q} .
$$


As mentioned above, $F \leqslant 1$ implies $h(x, t) \leqslant f(x, t)$. Therefore, it remains to prove that for all $t \geqslant 1$ and $x \in(0,2 \pi), f(x, t) \leqslant c h(x, t)$ for some fixed $c>0$. By the Markov property at time $t-1$, we have for $t>1$,

$$
h(x, t)=\mathbf{E}\left[\left(\Phi_{t-1}^{x}\right)^{b} h\left(Y_{t-1}^{x}, 1\right)\right]
$$

and similarly with $f$ replacing $h$ on both sides. Hence, it suffices to prove $\operatorname{ch}(x, 1) \geqslant$ $f(x, 1)$; i.e.,

$$
c \mathbf{E}\left[\left(\Phi_{1}^{x}\right)^{b} F\left(Y_{1}^{x}\right)\right] \geqslant \mathbf{E}\left[\left(\Phi_{1}^{x}\right)^{b}\right] .
$$

Let $\sigma_{y}=\sigma_{y}^{x}$ be the first time $s \geqslant 0$ such that $Y_{s}^{x}=y$, and if no such $s$ exists, set $\sigma_{y}=\infty$. By $(3.9), h(x, t)$ is a decreasing function of $t$. This and the strong Markov property give

$$
\mathbf{E}\left[F\left(Y_{1}^{x}\right) 1_{\left\{\sigma_{y}<1\right\}}\left(\Phi_{1}^{x}\right)^{b}\right] \geqslant h(y, 1) \mathbf{E}\left[1_{\left\{\sigma_{y}<1\right\}}\left(\Phi_{\sigma_{y}}^{x}\right)^{b}\right]
$$

Let

$$
a:=\min \left\{y>0: y-\cot \left(\frac{1}{2} y\right)=-2 \pi\right\}
$$

and consider the event

$$
\mathcal{A}:=\bigcap_{s \in[0,1]}\left\{Y_{s}^{x} \in(0, a)\right\}
$$

From (3.3) it then follows that on the event $\mathcal{A}, \sqrt{\kappa} B_{1} \leqslant a-\cot \left(\frac{1}{2} a\right)=-2 \pi$. Define the Brownian motion $\widetilde{B}$ on $[0,1]$ by $\widetilde{B}_{s}:=B_{s}-2 s B_{1}$, and define $\widetilde{Y}_{s}^{x}$ by the equation (3.3), but with $\widetilde{B}$ replacing $B$. Note that on $\mathcal{A}$ we have $B_{1}<0$, and hence

$$
\forall s \in[0,1], \quad \tilde{Y}_{s}^{x} \geqslant Y_{s}^{x}
$$

Moreover, given $\mathcal{A}$, there is a minimal $s_{0} \in[0,1]$ with $\widetilde{Y}_{s_{0}}^{x}=\pi$. Since $(3.11)$ holds on $\mathcal{A}$, it follows from (3.5) that

$$
\tilde{\Phi}_{s_{0}}^{x} \geqslant \Phi_{s_{0}}^{x} \geqslant \Phi_{1}^{x},
$$

where $\tilde{\Phi}$ is the analogue of $\Phi$ for the process $\tilde{Y}^{x}$. Since $\tilde{Y}^{x}$ has the same law as $Y^{x}$, with (3.10) this implies

$$
h(x, 1) \geqslant h(\pi, 1) \mathbf{E}\left[1_{\left\{\sigma_{\pi}<1\right\}}\left(\Phi_{\sigma_{\pi}}^{x}\right)^{b}\right] \geqslant h(\pi, 1) \mathbf{E}\left[1_{\mathcal{A}}\left(\Phi_{1}^{x}\right)^{b}\right] .
$$

The same proof gives this relation with $\mathcal{A}$ replaced by the event

$$
\mathcal{A}^{\prime}:=\bigcap_{s \in[0,1]}\left\{Y_{s}^{x} \in(2 \pi-a, 2 \pi)\right\} .
$$

However, for the event

$$
\mathcal{A}^{\prime \prime}:=\left\{\exists s \in[0,1]: Y_{s}^{x} \in[a, 2 \pi-a]\right\}
$$


we have

$$
h(x, 1) \geqslant h(a, 1) \mathbf{E}\left[1_{\mathcal{A}^{\prime \prime}}\left(\Phi_{1}^{x}\right)^{b}\right]
$$

Since $\mathcal{A} \cup \mathcal{A}^{\prime} \cup \mathcal{A}^{\prime \prime} \supset \mathcal{H}(x, 1)$ and $h(a, 1) \leqslant h(\pi, 1)$, we get

$$
h(x, 1) \geqslant \frac{1}{3} h(a, 1) f(x, 1) .
$$

This completes the proof of the lemma.

\subsection{Harmonic measure exponents}

By conformal invariance, the harmonic measure from 0 of $A_{t}$ in $D_{t}=\mathbf{U} \backslash K_{t}$ is $L_{t} / 2 \pi$, where $L_{t}$ is the length of the arc $g_{t}\left(A_{t}\right)$.

THEOREM 3.3. Suppose that $\kappa>0$ and $b \geqslant 1$. Then, when $t \rightarrow \infty$,

$$
\mathbf{E}\left[\left(L_{t}\right)^{b}\right] \asymp \exp (-\nu t)
$$

Proof. We have to relate the behavior of $\left|g_{t}^{\prime}\left(e^{i x}\right)\right|^{b}$, which we have studied above, to the behavior of

$$
\left(L_{t}\right)^{b}=\left(\int_{0}^{2 \pi}\left|g_{t}^{\prime}\left(e^{i x}\right)\right| d x\right)^{b}=\left(\int_{0}^{2 \pi} \Phi_{t}^{x} d x\right)^{b}
$$

where we set $\Phi_{t}^{x}=0$ if $\tau^{x} \leqslant t$. By convexity of the function $a \mapsto a^{b}$, it is clear that

$$
\begin{aligned}
\frac{1}{(2 \pi)^{b}} \mathbf{E}\left[\left(L_{t}\right)^{b}\right] & =\mathbf{E}\left[\left(\frac{1}{2 \pi} \int_{0}^{2 \pi} \Phi_{t}^{x} d x\right)^{b}\right] \leqslant \mathbf{E}\left[\frac{1}{2 \pi} \int_{0}^{2 \pi}\left(\Phi_{t}^{x}\right)^{b} d x\right] \\
& =\frac{1}{2 \pi} \int_{0}^{2 \pi} f(x, t) d x \leqslant c \exp (-\nu t),
\end{aligned}
$$

where we have used Lemma 3.2 for the last inequality. Consequently, we only need to prove the lower bound for $\mathbf{E}\left[\left(L_{t}\right)^{b}\right]$.

We will find constants $c_{1}, c_{2}>0$ and an event $\mathcal{U}_{t}^{*}$ such that

$$
\mathbf{E}\left[\left(\Phi_{t}^{\pi}\right)^{b} 1_{\mathcal{U}_{t}^{*}}\right] \geqslant c_{1} e^{-\nu t}
$$

and on the event $\mathcal{U}_{t}^{*}$,

$$
\left|\log \Phi_{t}^{\pi}-\log \Phi_{t}^{y}\right| \leqslant c_{2}, \quad \forall y \in\left[\pi, \pi+c_{1}\right]
$$

Then

$$
\mathbf{E}\left[\left(L_{t}\right)^{b}\right] \geqslant \mathbf{E}\left[1_{\mathcal{U}_{t}^{*}}\left(\int_{\pi}^{\pi+c_{1}} \Phi_{t}^{x} d x\right)^{b}\right] \geqslant\left(c_{1} e^{-c_{2}}\right)^{b} \mathbf{E}\left[1_{\mathcal{U}_{t}^{*}}\left(\Phi_{t}^{\pi}\right)^{b}\right] \geqslant c_{3} e^{-\nu t}
$$


which will prove the theorem.

Assume (with no loss of generality) that $t>3$, and let $t^{\prime}$ be the integer in $(t-2, t-1$ ] Define the event

$$
\mathcal{V}_{t}=\left\{\frac{1}{2} \pi \leqslant Y_{s}^{x} \leqslant \frac{3}{2} \pi, \forall s \in\left[t^{\prime}, t\right]\right\} .
$$

It follows from Lemma 3.2 that there is some constant $c_{4}>0$ such that

$$
\mathbf{E}\left[\left(\Phi_{t^{\prime}-1}^{x}\right)^{b} 1_{\left\{Y_{t^{\prime}-1}^{x} \in\left[c_{4}, 2 \pi-c_{4}\right]\right\}}\right] \geqslant c_{4} e^{-\nu t}
$$

This clearly implies

$$
\mathbf{E}\left[\left(\Phi_{t}^{\pi}\right)^{b} 1 \nu_{t}\right] \geqslant c_{5} e^{-\nu t}
$$

for some $c_{5}>0$.

Let $\mathcal{U}_{t}=\mathcal{U}_{t}(\alpha)$ be the event

$$
\mathcal{U}_{t}:=\left\{\alpha e^{-s / 8} \leqslant Y_{s}^{\pi} \leqslant 2 \pi-\alpha e^{-s / 8}, \forall s \in[0, t]\right\}
$$

We claim that for some $\alpha>0$, and every $t>3$,

$$
\mathbf{E}\left[\left(\Phi_{t}^{\pi}\right)^{b} 1_{\mathcal{U}_{t}} 1_{\mathcal{V}_{t}}\right] \geqslant \frac{1}{2} c_{5} e^{-\nu t}
$$

To prove this it suffices to show that for some $\alpha>0$,

$$
\mathbf{E}\left[\left(\Phi_{t}^{x}\right)^{b} 1_{\neg \mathcal{U}_{t}} 1_{\mathcal{V}_{t}}\right] \leqslant \frac{1}{2} c_{5} e^{-\nu t}
$$

For $u=0, \ldots, t^{\prime}-1$, and $\alpha \in\left(0, \frac{1}{5}\right)$, let $\mathcal{W}_{u}=\mathcal{W}_{u}(\alpha)$ be the event

$$
\mathcal{W}_{u}:=\left\{\alpha e^{-u / 8} \leqslant Y_{s}^{\pi} \leqslant 2 \pi-\alpha e^{-u / 8}, \forall s \in[u, u+1]\right\}
$$

Note that

$$
\bigcup_{u=0}^{t^{\prime}-1} \neg \mathcal{W}_{u} \supset \mathcal{V}_{t} \cap \neg \mathcal{U}_{t}
$$

Hence,

$$
\mathbf{E}\left[\left(\Phi_{t}^{x}\right)^{b} 1_{\neg \mathcal{U}_{t}} 1_{\mathcal{V}_{t}}\right] \leqslant \sum_{u=0}^{t^{\prime}-1} \mathbf{E}\left[\left(\Phi_{t}^{x}\right)^{b} 1_{\neg \mathcal{W}_{u}}\right]
$$

Note that for $u=0, \ldots, t^{\prime}-1$, the strong Markov property shows that

$$
\mathbf{E}\left[\left(\Phi_{t}^{x}\right)^{b} 1_{\neg \mathcal{W}_{u}} \mid \mathcal{F}_{u}\right] \leqslant\left(\Phi_{u}^{x}\right)^{b} f\left(\alpha e^{-u / 8}, t-u-1\right)
$$

(here $\mathcal{F}_{u}$ denotes the $\sigma$-field generated by $\left(Y_{s}, s \leqslant u\right)$ ). Hence, by Lemma 3.2,

$$
\mathbf{E}\left[\left(\Phi_{t}^{x}\right)^{b} 1_{\neg \mathcal{W}_{u}}\right] \leqslant c_{6} \alpha^{q} e^{-u q / 8} e^{-\nu t}
$$


Now (3.14) gives

$$
\mathbf{E}\left[\left(\Phi_{t}^{x}\right)^{b} 1_{\neg \mathcal{U}_{t}} 1_{\mathcal{V}_{t}}\right] \leqslant c_{7} \alpha^{q} e^{-\nu t}
$$

and hence by choosing $\alpha$ sufficiently small, we get (3.13) and therefore (3.12). Fix such an $\alpha \in\left(0, \frac{1}{5}\right)$, and let $\mathcal{U}_{t}^{*}:=\mathcal{U}_{t} \cap \mathcal{V}_{t}$.

Observe that (3.3) implies that if $0<x<y<2 \pi$,

$$
\partial_{t}\left(Y_{t}^{y}-Y_{t}^{x}\right)=\cot \left(\frac{1}{2} Y_{t}^{y}\right)-\cot \left(\frac{1}{2} Y_{t}^{x}\right) \leqslant-\frac{1}{2}\left(Y_{t}^{y}-Y_{t}^{x}\right)
$$

(since $\cot ^{\prime}(u) \leqslant-1$ in the range $u \in(0, \pi)$ ) as long as $t<\min \left\{\tau^{x}, \tau^{y}\right\}$, so that

$$
\left|Y_{t}^{y}-Y_{t}^{x}\right| \leqslant|x-y| e^{-t / 2}
$$

Let $y \in\left(\pi, \pi+\frac{1}{2} \alpha\right)$. Then

$$
0<Y_{s}^{y}-Y_{s}^{\pi} \leqslant e^{-s / 2}(y-\pi) \leqslant \frac{1}{2} \alpha e^{-s / 2}, \quad \forall s \leqslant \min \left\{\tau^{\pi}, \tau^{y}\right\} .
$$

On the event $\mathcal{U}_{t}^{*}$, we must therefore have $t<\min \left\{\tau^{x}, \tau^{y}\right\}$ and

$$
Y_{s}^{\pi}, Y_{s}^{y} \in\left[\frac{1}{2} \alpha e^{-s / 8}, 2 \pi-\frac{1}{2} \alpha e^{-s / 8}\right]
$$

for all $s \in[0, t]$. By (3.4), this shows that on the event $\mathcal{U}_{t}^{*}$, for all $s \leqslant t$,

$$
\begin{aligned}
\left|\partial_{s}\left(\log \Phi_{s}^{y}-\log \Phi_{s}^{\pi}\right)\right| & \leqslant\left|Y_{s}^{y}-Y_{s}^{\pi}\right| \max \left\{\frac{1}{2}\left|\partial_{x}\left(\sin ^{-2}\left(\frac{1}{2} x\right)\right)\right|: x \in\left[\frac{1}{2} \alpha e^{-s / 8}, 2 \pi-\frac{1}{2} \alpha e^{-s / 8}\right]\right\} \\
& \leqslant c_{7}\left|Y_{s}^{y}-Y_{s}^{\pi}\right| e^{3 s / 8}
\end{aligned}
$$

Now (3.15) gives

$$
\left|\partial_{s}\left(\log \Phi_{s}^{y}-\log \Phi_{s}^{\pi}\right)\right| \leqslant c_{8} e^{-s / 8} .
$$

Therefore, on the event $\mathcal{U}_{t}^{*}$,

$$
\left|\log \left(\Phi_{t}^{y} / \Phi_{t}^{\pi}\right)\right| \leqslant 8 c_{8}
$$

This completes the proof of the theorem.

\subsection{Extremal distance exponents}

Proof of Theorem 3.1. Let $\varrho(t):=\inf \left\{|z|: z \in K_{t}\right\}$. Recall that

$$
T(r)=\inf \{t: \varrho(t)=r\}
$$

$A_{t}=\partial \mathbf{U} \backslash \bar{K}_{t}$ and that $L_{t}$ is the length of the arc $g_{t}\left(A_{t}\right)$. Recall that the Schwarz lemma says that if $G: \mathbf{U} \rightarrow \mathbf{U}$ is analytic, then $\left|G^{\prime}(0)\right| \leqslant 1$, and the Koebe $\frac{1}{4}$-theorem says that 
if $G: \mathbf{U} \rightarrow \mathbf{C}$ is conformal with $G(0)=0$, then $G(\mathbf{U}) \supset \frac{1}{4}\left|G^{\prime}(0)\right| \mathbf{U}$. (See, e.g., [1].) Since $g_{t}^{\prime}(0)=\exp (t)$, applying the former to $z \mapsto g_{t}(\varrho(t) z)$ and the latter to $g_{t}^{-1}$ give

$$
\frac{1}{4} e^{-t} \leqslant \varrho(t) \leqslant e^{-t}
$$

In particular, if we fix the radius $r<\frac{1}{8}$ and define the deterministic times

$$
t^{\prime}=t^{\prime}(r):=\log \frac{1}{r}, \quad t=t(r):=\log \frac{1}{8 r}
$$

then

$$
t<T(r) \leqslant t^{\prime} \leqslant T\left(\frac{1}{4} r\right)
$$

and

$$
\varrho(t) \geqslant 2 r \geqslant r \geqslant \varrho\left(t^{\prime}\right) \geqslant \frac{1}{4} r \geqslant \frac{1}{8} r .
$$

In the following lines, $\ell\left(S, S^{\prime} ; U\right)$ will stand for the $\pi$-extremal distance between the sets $S$ and $S^{\prime}$ in $U$. Recall that $\mathfrak{L}(r)=\ell\left(C_{r}, C_{1} ; \mathbf{U} \backslash K_{T(r)}\right)$ and define $l_{r}=\ell\left(C_{r}, C_{1} ; \mathbf{U} \backslash K_{t(r)}\right)$. It follows from the above that

$$
l_{r} \leqslant \mathfrak{L}(r) \leqslant l_{r / 8} .
$$

Hence, it will be sufficient to study the asymptotic behavior of $\mathbf{E}\left[\exp \left(-b l_{r}\right)\right]$.

Note that $g_{t}: D_{t} \rightarrow \mathbf{U}$ is a conformal map defined on $D_{t} \supset 2 r \mathbf{U}$, that $g_{t}(0)=0$ and that $g_{t}^{\prime}(0)=1 / 8 r$. Hence, it follows immediately from the Schwarz lemma and the Koebe $\frac{1}{4}$-theorem that for any $r<\frac{1}{8}$,

$$
2^{-5} \mathbf{U} \subset g_{t}(r \mathbf{U}) \subset \frac{1}{2} \mathbf{U}
$$

Hence,

$$
\ell\left(C_{2^{-5}}, g_{t}\left(A_{t}\right) ; \mathbf{U}\right) \geqslant l_{r} \geqslant \ell\left(C_{1 / 2}, g_{t}\left(A_{t}\right) ; \mathbf{U}\right),
$$

and this implies easily that

$$
\exp \left(-l_{r}\right) \asymp L_{t(r)} .
$$

Theorem 3.1 now follows from Theorem 3.3 and (3.16).

\section{Properties of $\mathrm{SLE}_{6}$}

We now turn our attention towards specific properties of $\mathrm{SLE}_{6}$.

\subsection{Equivalence of chordal and radial $\mathrm{SLE}_{6}$}

The following result shows that chordal $\mathrm{SLE}_{6}$ and radial $\mathrm{SLE}_{6}$ are nearly the same process. (When $\kappa \neq 6$, a weaker form of equivalence holds.) A consequence of the equivalence 
of radial and chordal $\mathrm{SLE}_{6}$ is that radial $\mathrm{SLE}_{6}$ satisfies a restriction property, since in [27] a restriction property for chordal $\mathrm{SLE}_{6}$ has been established. The significance of the restriction property to the Brownian intersection exponents has been evident since [32].

THEOREM 4.1. Let $\theta \in \partial \mathbf{U} \backslash\{1\}$, and let $K_{t}$ be the hull of a radial $\mathrm{SLE}_{6}$-process in the unit disk $\mathbf{U}$ with driving process $\delta_{t}$ satisfying $\delta_{0}=\theta$. Set

$$
T:=\sup \left\{t \geqslant 0: 1 \notin \bar{K}_{t}\right\}
$$

Let $\widetilde{K}_{u}$ be the hull of a chordal $\mathrm{SLE}_{6}$-process in $\mathbf{U}$ starting also at $\theta$ and growing towards 1 , and let

$$
\widetilde{T}:=\sup \left\{u \geqslant 0: 0 \notin \widetilde{K}_{u}\right\} .
$$

Then, up to a random time change, the process $t \mapsto K_{t}$ restricted to $[0, T)$ has the same law as the process $u \mapsto \widetilde{K}_{u}$ restricted to $[0, \widetilde{T})$.

Note that $T$ (resp. $\widetilde{T}$ ) is the first time where $K_{t}$ (resp. $\widetilde{K}_{u}$ ) disconnects 0 from 1.

Proof. In order to point out where the assumption $\kappa=6$ is important, we let $\left(K_{t}, t \geqslant 0\right)$ and $\left(\widetilde{K}_{u}, u \geqslant 0\right)$ be $\mathrm{SLE}_{\kappa}$-processes, without fixing the value of $\kappa$ for the moment.

Let us first briefly recall (see, e.g., [27]) how $\widetilde{K}_{u}$ is defined. Let $\psi$ be the Möbius transformation that satisfies $\psi(\mathbf{U})=\mathbf{H}, \psi(1)=\infty, \psi(-1)=0$ and $\psi(0)=i$; i.e.,

$$
\psi(z)=i \frac{1+z}{1-z}
$$

Suppose that $u \mapsto \widetilde{B}_{u}$ is a real-valued Brownian motion such that $\sqrt{\kappa} \widetilde{B}_{0}=\psi\left(e^{i \theta}\right)$. For all $z \in \mathbf{U}$, define the function $\tilde{g}_{u}=\tilde{g}_{u}(z)$ such that $\tilde{g}_{0}(z)=\psi(z)$ and

$$
\partial_{u} \tilde{g}_{u}=\frac{2}{\tilde{g}_{u}-\sqrt{\kappa} \widetilde{B}_{u}} .
$$

This function is defined up to the (possibly infinite) time $\sigma_{z}$ where $\tilde{g}_{u}(z)$ hits $\sqrt{\kappa} \widetilde{B}_{u}$. Then, $\widetilde{K}_{u}$ is defined by $\widetilde{K}_{u}=\left\{z \in \mathbf{U}: \sigma_{z} \leqslant u\right\}$, so that $\tilde{g}_{u}$ is a conformal map from $\mathbf{U} \backslash \widetilde{K}_{u}$ onto the upper half-plane. This defines the process $\left(\widetilde{K}_{u}, u \geqslant 0\right)$ (the scaling property of Brownian motion shows that the choice of the conformal map $\psi$ only influences the law of $\left(\widetilde{K}_{u}\right)_{u \geqslant 0}$ via a time change).

We are now going to study the radial $\mathrm{SLE}_{\kappa}$. Let $g_{t}$ : $\mathbf{U} \backslash K_{t} \rightarrow \mathbf{U}$ be the conformal map normalized by $g_{t}(0)=0$ and $g_{t}^{\prime}(0)>0$. Recall that

$$
\partial_{t} g=g \frac{\delta+g}{\delta-g},
$$


where $\delta_{t}=\exp \left(i \sqrt{\kappa} B_{t}\right)$, and $B$ is Brownian motion on $\mathbf{R}$ with $\exp \left(i B_{0}\right)=\theta$. Let $\psi$ be the Möbius transformation as before, and define

$$
\begin{aligned}
e_{t} & :=g_{t}(1), \\
f_{t}(z) & :=\psi\left(g_{t}(z) / e_{t}\right), \\
\gamma_{t} & :=\psi\left(\delta_{t} / e_{t}\right) .
\end{aligned}
$$

These are well defined, as long as $t<T$. Note that $f_{t}$ is a conformal map from $\mathbf{U} \backslash K_{t}$ onto the upper half-plane, $f_{t}(1)=\infty$ and $\gamma_{t} \in \mathbf{R}$. From (4.1) it follows that

$$
\partial_{t} f=-\frac{\left(1+\gamma^{2}\right)\left(1+f^{2}\right)}{2(\gamma-f)} .
$$

Let

$$
\phi_{t}(z)=a(t) z+b(t)
$$

where

$$
a(0)=1, \quad \partial_{t} a=-\frac{1}{2}\left(1+\gamma^{2}\right) a
$$

and

$$
b(0)=0, \quad \partial_{t} b=-\frac{1}{2}\left(1+\gamma^{2}\right) a \gamma
$$

Set

$$
\begin{aligned}
h_{t} & :=\phi_{t} \circ f_{t}, \\
\beta_{t} & :=\phi_{t}(\gamma(t)) .
\end{aligned}
$$

Then (and this is the reason for the choice of the functions $a$ and $b$ )

$$
\partial_{t} h=-\frac{1}{2} a \frac{\left(1+\gamma^{2}\right)^{2}}{\gamma-f}=-\frac{\frac{1}{2}\left(1+\gamma^{2}\right)^{2} a^{2}}{\beta-h} .
$$

$h_{t}$ is also a conformal map from $\mathbf{U} \backslash K_{t}$ onto the upper half-plane with $h_{t}(1)=\infty$. Note also that $h_{0}(z)=\psi(z)$. We introduce a new time parameter $u=u(t)$ by setting

$$
\partial_{t} u=\frac{1}{4}\left(1+\gamma^{2}\right)^{2} a^{2}, \quad u(0)=0 .
$$

Then

$$
\frac{\partial h}{\partial u}=\frac{-2}{\beta-h} .
$$

Since this is the equation defining the chordal $\mathrm{SLE}_{6}$-process, it remains to show that $u \mapsto \beta_{t(u)} / \sqrt{\kappa}$ is Brownian motion (stopped at some random time). This is a direct but tedious application of Itô's formula:

$$
d \gamma_{t}=\frac{1}{2}\left(1+\gamma^{2}\right) \sqrt{\kappa} d B_{t}+\frac{1}{2} \gamma\left(1+\gamma^{2}\right)\left(\frac{1}{2} \kappa-1\right) d t
$$


and

$$
d \beta_{t}=\frac{1}{2}\left(1+\gamma^{2}\right) a\left(\sqrt{\kappa} d B_{t}+\left(-3+\frac{1}{2} \kappa\right) \gamma d t\right)
$$

This proves the claim, and establishes the theorem.

Note that when $\kappa \neq 6$, even though $u \mapsto \beta$ is not a local martingale, its law is absolutely continuous with respect to that of $\sqrt{\kappa}$ times a Brownian motion, as long as $\gamma$ and $u$ remain bounded. More precisely:

Proposition 4.2. Let $\left(K_{t}, t \geqslant 0\right),\left(\widetilde{K}_{u}, u \geqslant 0\right), T$ and $\widetilde{T}$ be defined just as in Theorem 4.1, except that they are $\mathrm{SLE}_{\kappa}$ with general $\kappa>0$. There exist two non-decreasing families of stopping times, $\left(T_{n}, n \geqslant 1\right)$ and $\left(\widetilde{T}_{n}, n \geqslant 1\right)$, such that almost surely $T_{n} \rightarrow T$ and $\widetilde{T}_{n} \rightarrow \widetilde{T}$ when $n \rightarrow \infty$, and such that for each $n \geqslant 1$, the laws of $\left(K_{t}, t \in\left[0, T_{n}\right]\right)$ and $\left(\widetilde{K}_{u}, u \in\left[0, \widetilde{T}_{n}\right]\right)$ are equivalent (in the sense that they have a positive density with respect to each other) modulo increasing time change.

Proof. It suffices to take

$$
T_{n}=\min \left\{n, \inf \left\{t>0:\left|\delta_{t}-e_{t}\right|<1 / n\right\}\right\}
$$

Then, it is easy to see that before $T_{n},|\gamma|$ remains bounded, $a$ is bounded away from 0 (note also that $a \leqslant 1$ always), so that $t / u$ is bounded and bounded away from 0 . Hence, $u\left(T_{n}\right)$ is also bounded (since $T_{n} \leqslant n$ ).

It now follows directly from Girsanov's theorem (see, e.g., [38]) that the law of $(\beta(u) / \sqrt{\kappa})_{u \leqslant u\left(T_{n}\right)}$ is equivalent to that of Brownian motion up to some (bounded) stopping time, and Proposition 4.2 follows.

\subsection{The crossing exponent for $\mathrm{SLE}_{6}$}

In this section, we are going to study the probability that chordal $\mathrm{SLE}_{6}$ started at some point on the left-hand side of a rectangle crosses the rectangle from the left to the right without touching the upper and lower boundaries of the rectangle. As we shall see, the estimate obtained is a direct consequence of Cardy's formula for SLE 6 proved in [27].

The notation turns out to be simpler when considering crossings of a quadrilateral in the unit disk, which is equivalent to the rectangle case by conformal invariance. We now describe the setup more precisely. Recall that when $w, w^{\prime} \in \partial \mathbf{U}$, the counterclockwise 


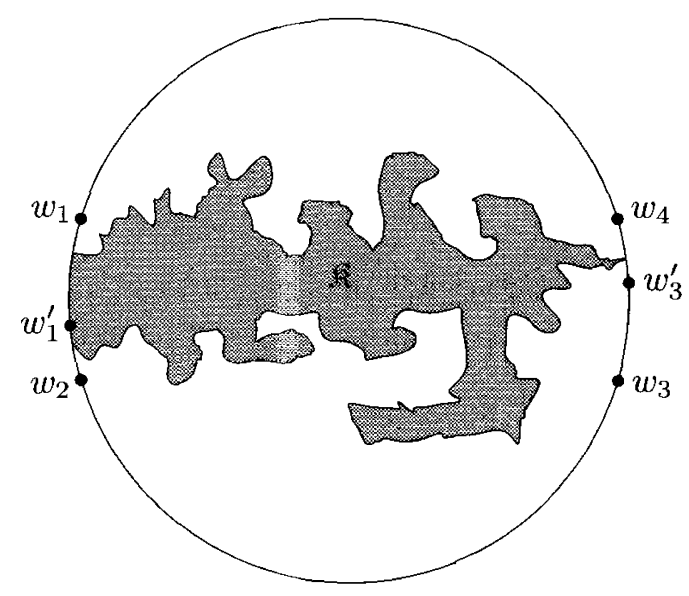

Fig. 4.1. A successful crossing.

arc from $w$ to $w^{\prime}$ is denoted $a\left(w, w^{\prime}\right)$. Let $\theta \in\left(0, \frac{1}{2} \pi\right), \alpha \in(-1,1)$, and define the points

$$
\begin{aligned}
& w_{1}:=\exp (i(\pi-\theta)), \\
& w_{1}^{\prime}:=\exp (i(\pi+\alpha \theta)), \\
& w_{2}:=\exp (i(\pi+\theta)), \\
& w_{3}:=\exp (-i \theta), \\
& w_{4}:=\exp (i \theta),
\end{aligned}
$$

and note that they appear in counterclockwise order on $\partial \mathbf{U}$. See Figure 4.1. Let $w_{3}^{\prime}$ be any point in $a\left(w_{3}, w_{4}\right)$. Consider a chordal $\mathrm{SLE}_{6}$ in $\mathbf{U}$ started from $w_{1}^{\prime}$ and growing towards $w_{3}^{\prime}$. Let $K_{t}$ be the hull, and let $T$ be the first time $t$ such that $\bar{K}_{t}$ intersects the $\operatorname{arc} a\left(w_{3}, w_{4}\right)$. Set

$$
\mathfrak{K}:=\bigcup_{t<T} \bar{K}_{t} .
$$

As shown in [27], the restriction property for $\mathrm{SLE}_{6}$ shows that up to a monotone time change, the law of the process $\left(K_{t}\right)_{t<T}$ does not depend on the choice of $w_{3}^{\prime}$. Since we use the restriction property, the result derived in this subsection is specific to $\kappa=6$.

We are interested in the event

$$
\mathcal{E}:=\left\{\mathfrak{K} \cap\left(a\left(w_{2}, w_{3}\right) \cup a\left(w_{4}, w_{1}\right)\right)=\varnothing\right\} .
$$

Lemma 4.3. Suppose that $\alpha_{0} \in(0,1)$ is fixed. When $\theta \searrow 0$,

$$
\mathbf{P}[\mathcal{E}] \asymp \theta^{2},
$$

and this estimate is uniform for $\alpha \in\left(-\alpha_{0}, \alpha_{0}\right)$. Moreover, when $\theta \searrow 0$,

$$
\max _{\alpha \in(-1,1)} \mathbf{P}[\mathcal{E}] \asymp \theta^{2} \text {. }
$$


Recall also that $\theta^{2} \sim \exp (-\ell)$ as $\theta \searrow 0$, where $\ell=\ell\left(a\left(w_{1}, w_{2}\right), a\left(w_{3}, w_{4}\right) ; \mathbf{U}\right)$ denotes the $\pi$-extremal distance between $a\left(w_{1}, w_{2}\right)$ and $a\left(w_{3}, w_{4}\right)$ in $\mathbf{U}$.

The lemma is hardly surprising. Suppose for a moment that we take $w_{1}^{\prime}=-1$, and let $T^{\prime}$ be the first time such that $\bar{K}_{t}$ intersects $a(-i, i)$. Let $z$ be the point in $a(-i, i)$ which is on the boundary of $\bigcup_{t<T^{\prime}} K_{t}$. It is then easy to believe that the probability density for the location of $z$ should be bounded away from 0 and infinity in every compact subset of $a(-i, i)$. By conformal invariance, this implies the first part of the lemma for the case $\alpha=0$. Although it should not be too hard to prove the lemma with some general arguments such as these, we find it easier to rely on the more refined results from [27].

Proof. Define the events

$$
\begin{aligned}
& \mathcal{E}^{\prime}=\left\{\bar{K}_{t} \text { hits } a\left(w_{2}, w_{4}\right) \text { before } a\left(w_{4}, w_{1}\right)\right\} \\
& \mathcal{E}^{\prime \prime}=\left\{\bar{K}_{t} \text { hits } a\left(w_{2}, w_{3}\right) \text { before } a\left(w_{3}, w_{1}\right)\right\}
\end{aligned}
$$

Note that $\mathcal{E}^{\prime \prime} \subset \mathcal{E}^{\prime}$, and that $\mathcal{E}=\mathcal{E}^{\prime} \backslash \mathcal{E}^{\prime \prime}$. Cardy's formula for SLE 6 derived in [27] (Theorem 3.2 in the case where $b=0)$ gives the exact value of $\mathbf{P}\left(\mathcal{E}^{\prime}\right)$ and $\mathbf{P}\left(\mathcal{E}^{\prime \prime}\right)$, as follows. Define the cross ratios

$$
c^{\prime}:=\frac{\left(w_{1}-w_{1}^{\prime}\right)\left(w_{4}-w_{2}\right)}{\left(w_{4}-w_{1}^{\prime}\right)\left(w_{1}-w_{2}\right)}, \quad c^{\prime \prime}:=\frac{\left(w_{1}-w_{1}^{\prime}\right)\left(w_{3}-w_{2}\right)}{\left(w_{3}-w_{1}^{\prime}\right)\left(w_{1}-w_{2}\right)}
$$

and set

$$
G(x):={ }_{2} F_{1}\left(\frac{1}{3}, \frac{2}{3}, \frac{4}{3} ; x\right) \frac{\sqrt{\pi}}{2^{1 / 3} \Gamma\left(\frac{1}{3}\right) \Gamma\left(\frac{7}{6}\right)} x^{1 / 3}
$$

(where ${ }_{2} F_{1}$ denotes the hypergeometric function). Then

$$
\mathbf{P}\left[\mathcal{E}^{\prime}\right]=G\left(c^{\prime}\right), \quad \mathbf{P}\left[\mathcal{E}^{\prime \prime}\right]=G\left(c^{\prime \prime}\right), \quad \mathbf{P}[\mathcal{E}]=G\left(c^{\prime}\right)-G\left(c^{\prime \prime}\right)
$$

(To compute $\mathbf{P}\left[\mathcal{E}^{\prime}\right]$, we view $K_{t}$ as an $\mathrm{SLE}_{6}$ from $w_{1}^{\prime}$ to $w_{4}$, while to compute $\mathbf{P}\left[\mathcal{E}^{\prime \prime}\right]$, we view $K_{t}$ as an $\mathrm{SLE}_{6}$ from $w_{1}^{\prime}$ to $w_{3}$. As remarked above, the choice of $w_{3}^{\prime} \in a\left(w_{3}, w_{4}\right)$ does not matter.) Note that

$$
c^{\prime}=\cot \theta \tan \left(\frac{1}{2}(1+\alpha) \theta\right), \quad c^{\prime \prime}=\frac{\sin \left(\frac{1}{2}(1+\alpha) \theta\right)}{\sin \theta \cos \left(\frac{1}{2}(1-\alpha) \theta\right)}
$$

Both $c^{\prime}$ and $c^{\prime \prime}$ converge to $\frac{1}{2}(1+\alpha)$ when $\theta \searrow 0$ and $c^{\prime}-c^{\prime \prime} \sim \frac{1}{4}\left(1-\alpha^{2}\right) \theta^{2}$. Since $G^{\prime}(x)=$ $\left(\frac{1}{3} \sqrt{\pi}\right) 2^{-1 / 3} \Gamma\left(\frac{1}{3}\right)^{-1} \Gamma\left(\frac{7}{6}\right)^{-1}((1-x) x)^{-2 / 3}$, it follows that

$$
\mathbf{P}[\mathcal{E}]=G\left(c^{\prime}\right)-G\left(c^{\prime \prime}\right) \sim\left(\frac{1}{6} \sqrt{\pi}\right) \Gamma\left(\frac{1}{3}\right)^{-1} \Gamma\left(\frac{7}{6}\right)^{-1}\left(1-\alpha^{2}\right)^{1 / 3} \theta^{2},
$$

as $\theta \searrow 0$, and the lemma follows. 


\section{Brownian intersection exponents}

\subsection{Definitions and statement of results}

This section begins with a review of the definitions and some general facts concerning intersection exponents between planar Brownian paths, and proceeds with a statement of some theorems. For more details concerning the background results on intersection exponents, as well as some references to the literature, see [31], [32].

Suppose that $n+m$ independent Brownian motions $B^{1}, \ldots, B^{n}$ and $B^{\prime 1}, \ldots, B^{\prime m}$ are started from points $B^{1}(0)=\ldots=B^{n}(0)=i$ and $B^{\prime 1}(0)=\ldots=B^{\prime m}(0)=1$ in the complex plane, and let $\mathfrak{B}_{r}^{j}, \mathfrak{B}_{r}^{\prime k}$ denote the traces (i.e., images) of the paths up to the first time they reach the circle $C_{r}$. Consider the probability $f_{n, m}(r)$ that the $\mathfrak{B}$-traces do not intersect the $\mathfrak{B}^{\prime}$-traces, i.e.,

$$
f_{n, m}(r):=\mathbf{P}\left[\left(\bigcup_{j=1}^{n} \mathfrak{B}_{r}^{j}\right) \cap\left(\bigcup_{l=1}^{m} \mathfrak{B}_{r}^{l}\right)=\varnothing\right] .
$$

It is easy to see that as $r \rightarrow \infty$ this probability decays like a power law; the $(n, m)$ intersection exponent $\xi(n, m)$ is defined by

$$
f_{n, m}(r)=r^{-\xi(n, m)+o(1)}, \quad r \rightarrow \infty .
$$

We call $\xi(n, m)$ the intersection exponent between one packet of $n$ Brownian motions and one packet of $m$ Brownian motions. It is easy to see (e.g. [31]) that the exponent $\zeta(n, m)$ described in the introduction is equal to $\frac{1}{2} \xi(n, m)$.

By using the conformal map $z \mapsto 1 / z$ and invariance of planar Brownian motion under conformal mapping, it is clear that

$$
f_{n, m}(r)=f_{n, m}(1 / r)
$$

so that the exponents also measure the decay of $f_{n, m}$ when $r \searrow 0$.

Similarly, one can define corresponding probabilities for intersection exponents in a half-plane,

$$
\tilde{f}_{n, m}(r):=\mathbf{P}\left[\left(\bigcup_{j=1}^{n} \mathfrak{B}_{r}^{j}\right) \cap\left(\bigcup_{l=1}^{m} \mathfrak{B}_{r}^{\prime l}\right)=\varnothing \text { and }\left(\bigcup_{j=1}^{n} \mathfrak{B}_{r}^{j}\right) \cup\left(\bigcup_{l=1}^{m} \mathfrak{B}_{r}^{\prime l}\right) \subset H\right]
$$

where $H$ is some half-plane through the origin containing 1 and $i .\left(\tilde{f}_{n, m}(r)\right.$ does depend on $H$.) In plain words, we are looking at the probability that all Brownian motions stay in the half-plane $H$ and that all the $\mathfrak{B}$-traces avoid all the $\mathfrak{B}^{\prime}$-traces. It is also easy to see that there exists a $\tilde{\xi}(n, m)$ (which does not depend on $H$ ) such that

$$
\tilde{f}_{n, m}(r)=r^{-\tilde{\xi}(n, m)+o(1)}
$$

when $r \rightarrow \infty$. 
These intersection exponents can be generalized in a number of ways. For example, set

$$
Z_{r}:=\mathbf{P}\left[\mathfrak{B}_{r}^{\prime 1} \cap \bigcup_{j=1}^{n} \mathfrak{B}_{r}^{j}=\varnothing \mid \bigcup_{j=1}^{n} \mathfrak{B}_{r}^{j}\right]
$$

Then $f_{n, m}(r)=\mathbf{E}\left[Z_{r}^{m}\right]$, and define $\xi(n, \lambda)$ for all $\lambda>0$ by the relation

$$
\mathbf{E}\left[Z_{r}^{\lambda}\right] \approx r^{-\xi(n, \lambda)}, \quad r \rightarrow \infty .
$$

It has been proved by Lawler [24] that in fact

$$
\mathbf{E}\left[Z_{r}^{\lambda}\right] \asymp r^{-\xi(2, \lambda)} .
$$

The same proof, with minor notational modifications, applies to the other exponents as well. See also [30] for a new self-contained proof of this result.

One can also define the exponents $\xi\left(n_{1}, n_{2}, \ldots, n_{k}\right)$ and $\tilde{\xi}\left(n_{1}, n_{2}, \ldots, n_{k}\right)$ describing the probability of non-intersection of $k$ packets of Brownian motions. In fact, [31] proves that there is a natural and rigorous way to generalize these definitions to the case where the numbers $n_{j}$ are positive reals (i.e., not required to be integers). For the definition of $\xi\left(\lambda_{1}, \ldots, \lambda_{k}\right)$ one only needs to assume that at least two of the numbers $\lambda_{1}, \ldots, \lambda_{k}$ are at least 1 , and for $\tilde{\xi}$ even this assumption is not necessary. What makes these extended definitions natural is that they are uniquely determined by certain identities and relations. For one, the functions $\xi$ and $\tilde{\xi}$ are invariant under permutations of their arguments. Moreover, they satisfy the so-called cascade relations [31]: for any $1 \leqslant q \leqslant m-1$ and $\left(\lambda_{1}, \ldots, \lambda_{m}\right)$ such that $\lambda_{1} \geqslant 1$ and $\max \left\{\lambda_{2}, \ldots, \lambda_{m}\right\} \geqslant 1$,

$$
\xi\left(\lambda_{1}, \ldots, \lambda_{m}\right)=\xi\left(\lambda_{1}, \ldots, \lambda_{q}, \tilde{\xi}\left(\lambda_{q+1}, \ldots, \lambda_{m}\right)\right) .
$$

In [31] it was also established that the cascade relations imply the existence of a continuous increasing function $\eta:[\tilde{\xi}(1,1), \infty) \rightarrow[\xi(1,1), \infty)$ such that for all $\left(\lambda_{1}, \ldots, \lambda_{m}\right) \in \mathbf{R}_{+}^{m}$ such that at least two of the $\lambda_{j}$ 's are at least 1 ,

$$
\xi\left(\lambda_{1}, \ldots, \lambda_{m}\right)=\eta\left(\tilde{\xi}\left(\lambda_{1}, \ldots, \lambda_{m}\right)\right) .
$$

In [27], we have determined the exact value of exponents $\tilde{\xi}\left(\lambda_{1}, \ldots, \lambda_{m}\right)$ for a certain class of numbers $\left(\lambda_{1}, \ldots, \lambda_{m}\right)$; namely, for all $m \geqslant 2$, and all $\left(\lambda_{1}, \ldots, \lambda_{m}\right) \in$ $\left\{\frac{1}{6} l(l+1): l \in \mathbf{N}\right\}^{m-1} \times \mathbf{R}_{+}$,

$$
\tilde{\xi}\left(\lambda_{1}, \ldots, \lambda_{m}\right)=\frac{1}{24}\left(\left(\sqrt{24 \lambda_{1}+1}+\ldots+\sqrt{24 \lambda_{m}+1}-(m-1)\right)^{2}-1\right) .
$$

In particular,

$$
\begin{aligned}
\tilde{\xi}(1,1, \lambda) & =\frac{1}{24}\left((8+\sqrt{24 \lambda+1})^{2}-1\right), \\
\tilde{\xi}(1,1,1, \lambda) & =\frac{1}{24}\left((12+\sqrt{24 \lambda+1})^{2}-1\right) .
\end{aligned}
$$

In the next section, we will prove the following two results: 
THEOREM 5.1.

$$
\xi(1,1)=\frac{5}{4}
$$

THEOREM 5.2. For every $\lambda \geqslant 0$,

$$
\xi(1,1,1, \lambda)=\frac{1}{48}\left((11+\sqrt{24 \lambda+1})^{2}-4\right)
$$

Let us now see what these two results directly imply:

THEOREM 5.3. For all $x \geqslant 7$,

$$
\eta(x)=\frac{1}{48}\left((\sqrt{24 x+1}-1)^{2}-4\right)
$$

Moreover, for all $m \geqslant 2$ and for all $\left(\lambda_{1}, \ldots, \lambda_{m}\right) \in\left\{\frac{1}{6} l(l+1): l \in \mathbf{N}\right\}^{m-1} \times \mathbf{R}_{+}$,

$$
\xi\left(\lambda_{1}, \ldots, \lambda_{m}\right)=\frac{1}{48}\left(\left(\sqrt{24 \lambda_{1}+1}+\ldots+\sqrt{24 \lambda_{m}+1}-m\right)^{2}-4\right),
$$

provided that at least two of the numbers $\lambda_{1}, \ldots, \lambda_{m}$ are at least 1 , and the right-hand side of $(5.9)$ is at least $\frac{35}{12}$.

In particular, for all $\lambda \geqslant \frac{10}{3}$ and $\lambda^{\prime} \geqslant 2$,

$$
\begin{gathered}
\xi(1, \lambda)=\frac{1}{48}\left((3+\sqrt{24 \lambda+1})^{2}-4\right), \\
\xi\left(2, \lambda^{\prime}\right)=\frac{1}{48}\left(\left(5+\sqrt{24 \lambda^{\prime}+1}\right)^{2}-4\right),
\end{gathered}
$$

and for all integers $m \geqslant 2$ (using (5.6) for the case $m=2$ ),

$$
\xi\left(1^{\otimes m}\right)=\frac{1}{12}\left(4 m^{2}-1\right)
$$

where $1^{\otimes m}:=(1, \ldots, 1) \in \mathbf{N}^{m}$.

Proof of Theorem 5.3 (assuming (5.6) and (5.7)). Combining (5.5), (5.7) and (5.2) gives (5.8). Hence, we get (5.9) from (5.3), (5.2) and (5.8).

Proof of Theorems 1.1 and 1.2 (assuming (5.6) and (5.7)). In view of the fact that the time exponents differ by a factor of 2 from the space exponents, the theorems follow from Theorem 5.3.

Remark. In [28], we will show that (5.3) holds for all $\left(\lambda_{1}, \ldots, \lambda_{m}\right) \in \mathbf{R}_{+}^{m}$. The proofs in the present paper can then be very easily adapted to show that (5.9) holds for all $\left(\lambda_{1}, \ldots, \lambda_{m}\right) \in \mathbf{R}_{+}^{m}$ such that at least two of the $\lambda_{j}$ 's are at least 1 . 


\subsection{Excursion measures}

In [31], [32] a characterization of the intersection exponents was given in terms of excursions. The Brownian excursion measures are natural and interesting objects. The utility of the excursion measures in [31], [32] arises from the fact that in the context of excursion measures, one can study Brownian motions without specifying the starting point. This significantly simplifies some arguments and estimates.

Roughly speaking, Brownian excursions in a domain $D$ are Brownian motions started on the boundary, conditioned to immediately enter $D$, and stopped upon leaving. Since Brownian excursions stay in the domain $D$, conformal transformations of $D$ can be applied to the excursions.

Let us now describe these Brownian excursions more precisely. For any bounded simply-connected open domain $D$, there exists a Brownian excursion measure $\mu_{D}$ in $D$. This is an infinite measure on the set of paths $(B(t), t \leqslant \tau)$ in $D$ such that $B(0, \tau) \subset D$ and $B(0), B(\tau) \in \partial D$ (these endpoints can be viewed as prime ends if necessary). $x_{s}:=B(0)$ and $x_{e}:=B(\tau)$ will denote the starting point and terminal point of the excursion. In this discussion, we will identify two paths (two excursions) when one is obtained by an increasing time change of the other.

One possible definition of $\mu_{D}$ is the following. Consider first $D=\mathbf{U}$, the unit disc. For every $s \in(0,1)$ let $P^{s}$ be the law of a Brownian motion started uniformly on the circle of radius $s$, and stopped when it exits $\mathbf{U}$ (modulo continuous increasing time change). Since $z \mapsto \log |z|$ is harmonic, for any $r \in(0, s)$,

$$
P^{s}\left[B \text { hits } C_{r}\right]=\frac{\log (1 / s)}{\log (1 / r)} .
$$

Set

$$
\mu_{\mathbf{U}}:=\lim _{s \nearrow 1} \frac{2 \pi}{\log (1 / s)} P^{s},
$$

as a weak limit. Note that the $\mu_{\mathrm{U}}$-measure of the set of paths that hit the circle $C_{r}$ is $2 \pi / \log (1 / r)$.

One can then check that for any Möbius transformation $\phi$ from $\mathbf{U}$ onto $\mathbf{U}$, $\phi\left(\mu_{\mathbf{U}}\right)=\mu_{\mathbf{U}}$. This makes it possible to extend the definition of $\mu_{D}$ to any simplyconnected domain $D$, by conformal invariance. These Brownian excursions also have a "restriction" property [32], which is a result of the fact that the Brownian paths only feel the boundary of $D$ when they hit it (and then stop).

In [27], we made an extensive use of the Brownian excursion measure in rectangles $R_{L}=(0, L) \times(0, \pi)$. It is easy to see that the measure $\mu_{R_{L}}$, restricted to those excursions with starting point on the left-hand side of the rectangle $[0, i \pi]$, is obtained as the limit when $s \rightarrow 0$ of $\pi s^{-1} P_{L}^{s}$, where $P_{L}^{s}$ is the law of a Brownian motion with uniform starting 
point on $[s, s+i \pi]$ which is stopped when it exits $R_{L}$. In particular, this leads to the following result:

Lemma 5.4. Let $\mathcal{E}_{L}$ denote the event that the Brownian excursion $B$ in $R_{L}$ crosses the rectangle from the left to the right (i.e., $x_{s} \in(0, i \pi)$ and $\left.x_{e} \in(L, L+i \pi)\right)$. Then, when $L \rightarrow \infty$,

$$
\mu_{R_{L}}\left[\mathcal{E}_{L}\right] \asymp e^{-L}
$$

Proof. Let $h_{z}$ denote harmonic measure from $z$ on $\partial R_{L}$, where $z \in R_{L}$. Since $\operatorname{Im}(\exp z)$ is a harmonic function, it easily follows that for all $L>1$ and $z \in(1,1+i \pi)$,

$$
\sin \left(\frac{1}{4} \pi\right) h_{z}\left(\left[L+\frac{1}{4} i \pi, L+\frac{3}{4} i \pi\right]\right) \leqslant e^{-L} \leqslant \frac{h_{z}([L, L+i \pi])}{\operatorname{Im}(\exp (z))-1}
$$

It is easy to verify (e.g., by conformal invariance or by a reflection argument) that there is a constant $c$ such that $h_{z}([L, L+i \pi]) \leqslant c h_{z}\left(\left[L+\frac{1}{4} i \pi, L+\frac{3}{4} i \pi\right]\right)$ holds for all $L>2$ and $z \in(1,1+i \pi)$. Hence, for such $L$ and $z$,

$$
c_{1} h_{z}([L, L+i \pi]) \leqslant e^{-L} \leqslant c_{2} h_{z}([L, L+i \pi]) 1_{\left\{\operatorname{Im}(z) \in\left[\frac{1}{4} \pi, \frac{3}{4} \pi\right]\right\}},
$$

with some constants $c_{1}, c_{2}>0$. Since the $\mu_{R_{L}}$-measure of the excursions which reach the line $\{\operatorname{Re}(z)=1\}$ does not depend on $L$, and from these a fixed proportion first hit $\{\operatorname{Re}(z)=1\}$ in $\left[1+\frac{1}{4} \pi, 1+\frac{3}{4} \pi\right],(5.13)$ now follows from the Markov property, which is valid for the excursion measures.

Similarly, one can define Brownian excursions in non-simply-connected domains. For instance, consider the annulus $A(r, 1)$ bounded between the circles $C_{r}$ and $C_{1}$ (where $r \in(0,1))$. Rather than defining the excursion in $A(r, 1)$ directly, we base the definition on the excursions in $\mathbf{U}$. If $\gamma$ is a path, let $\Psi_{r}(\gamma)$ be the initial segment of $\gamma$, until the first hit of $C_{r}$, or all of $\gamma$, if $\gamma$ does not hit $C_{r}$. Now set $\mu_{1}^{r}:=\Psi_{r}\left(\mu_{\mathrm{U}}\right)$. This will be called the Brownian excursion measure on $A(r, 1)$ for excursions started on $C$. It is clear that $\mu_{\mathbf{U}}=\lim _{r \searrow 0} \mu_{1}^{r}$.

The measures $\mu_{D}$ and $\mu_{1}^{r}$ are also related by restriction and conformal invariance. Suppose that $O$ is a simply-connected subset of $A(r, 1)$ such that each of the sets $\bar{O} \cap C_{r}$ and $\bar{O} \cap C$ is an arc of positive length. Let $L$ denote the $\pi$-extremal distance between these two $\operatorname{arcs}$ in $O$, and let $\phi$ denote the conformal map from $O$ onto $R_{L}=(0, L) \times(0, \pi)$ such that $\bar{O} \cap C$ corresponds to $(0, i \pi)$ and $\bar{O} \cap C_{r}$ corresponds to $(L, L+i \pi)$ under $\phi$.

Let $\hat{\mathcal{E}}_{1}$ be the set of paths starting in $C$ that reach $C_{r}$ without exiting $\bar{O}$. Consider the image under $\phi$ of the measure $\mu_{1}^{r}$ restricted to $\hat{\mathcal{E}}_{1}$. Then (up to time change) the 
image measure is exactly $\mu_{R_{L}}$ restricted to the set of excursions that cross the rectangle from the left to the right. (5.13) therefore shows that when $L \rightarrow \infty$,

$$
\mu_{1}^{r}\left[\hat{\mathcal{E}}_{1}\right] \asymp \exp (-L) .
$$

This may also be easily verified directly.

The mapping $z \mapsto r / z$ maps $A(r, 1)$ conformally onto itself. Let $\mu_{r}^{1}$ be the image of $\mu_{1}^{r}$ under this map; this is a measure on Brownian excursions started on $C_{r}$ and stopped upon leaving $A(r, 1)$. By symmetry, it follows that $\mu_{r}^{1}\left[\hat{\mathcal{E}}_{1}\right]=\mu_{1}^{r}\left[\hat{\mathcal{E}}_{1}\right] \asymp \exp (-L)$ when $L \rightarrow \infty$.

Although we will not use this here, it is worthwhile to note that the measures $\mu_{r}^{1}$ and $\mu_{1}^{r}$ agree on the set of paths crossing the annulus, up to time reversal of the path.

\subsection{Exponents and excursions}

We now describe the intersection exponents in terms of excursions (referring to [31], [32] for the proofs). Let $(B(t), t \leqslant \tau)$ be an excursion in $R_{L}$, and, as above, let

$$
\mathcal{E}_{L}:=\left\{\operatorname{Re}\left(x_{s}\right)=0 \text { and } \operatorname{Re}\left(x_{e}\right)=L\right\}
$$

be the event that $B$ crosses $R_{L}$ from left to right. Let $\mathfrak{B}$ denote the image of $B$. When $\mathcal{E}_{L}$ holds, let $O_{B}^{+}$be the component of $R_{L} \backslash \mathfrak{B}$ above $\mathfrak{B}$, and let $O_{B}^{-}$be the component of $R_{L} \backslash \mathfrak{B}$ below $\mathfrak{B}$. Let $\mathfrak{L}_{B}^{-}$(resp. $\mathfrak{L}_{B}^{+}$) denote the $\pi$-extremal distance between $\left[0, x_{s}\right]$ and $\left[L, x_{e}\right]$ in $O_{B}^{-}$(resp. $\left[x_{s}, i \pi\right]$ and $\left[x_{e}, L+i \pi\right]$ in $O_{B}^{+}$). (We use script fonts for these $\mathfrak{L}$ to indicate that they are random variables.) Then, for any $\alpha \geqslant 0$ and $\alpha^{\prime} \geqslant 0$, the exponent $\tilde{\xi}\left(\alpha, 1, \alpha^{\prime}\right)=\tilde{\xi}\left(1, \tilde{\xi}\left(\alpha, \alpha^{\prime}\right)\right)$ is characterized by

$$
\int_{\mathcal{E}_{L}} \exp \left(-\alpha \mathfrak{L}_{B}^{+}-\alpha^{\prime} \mathfrak{L}_{B}^{-}\right) d \mu_{R_{L}}(B)=\exp \left(-\tilde{\xi}\left(\alpha^{\prime}, 1, \alpha\right) L+o(L)\right)
$$

as $L \rightarrow \infty$.

Let $\mathcal{E}_{L}^{2}$ be the set of pairs of paths $\left(B, B^{\prime}\right) \in \mathcal{E}_{L} \times \mathcal{E}_{L}$ such that the trace $\mathfrak{B}^{\prime}$ of $B^{\prime}$ is contained in $O_{B}^{-}$. It follows from (5.15), Lemma 5.4 and conformal invariance of the excursion measures that

$$
\int_{\mathcal{E}_{L}^{2}} \exp \left(-\alpha \mathfrak{L}_{B}^{+}\right) d \mu_{R_{L}}(B) d \mu_{R_{L}}\left(B^{\prime}\right)=\exp (-\tilde{\xi}(1,1, \alpha) L+o(L))
$$

as $L \rightarrow \infty$. On $\mathcal{E}_{L}^{2}$, let $\mathfrak{L}_{B^{\prime}}^{B}$ be the $\pi$-extremal distance from $[0, i \pi]$ to $[L, L+i \pi]$ in the domain between $\mathfrak{B}$ and $\mathfrak{B}^{\prime}$. Given $B^{\prime}$, it is clear by conformal invariance and the restriction property of the excursion measure that $1_{\mathcal{E}_{L}^{2}} \mathfrak{L}_{B^{\prime}}^{B}$ has the same law as $1_{\mathcal{E}_{L}^{2}} \mathfrak{L}_{B}^{+}$. Consequently, (5.16) gives

$$
\int_{\mathcal{E}_{L}^{2}} \exp \left(-\alpha \mathfrak{L}_{B^{\prime}}^{B}\right) d \mu_{R_{L}}(B) d \mu_{R_{L}}\left(B^{\prime}\right)=\exp (-\tilde{\xi}(1,1, \alpha) L+o(L)) .
$$


Similarly, one can characterize the exponents $\xi$ in terms of the excursion measure $\mu_{r}^{1}$. For any $r<1$, consider two independent excursions $B$ and $B^{\prime}$ of the annulus $A(r, 1)$. Define the events

$$
\begin{aligned}
& \mathcal{E}=\mathcal{E}(r):=\left\{\mathfrak{B} \text { crosses the annulus without separating } C_{r} \text { from } C\right\} \\
& \tilde{\mathcal{E}}=\tilde{\mathcal{E}}(r):=\left\{\mathfrak{B} \text { and } \mathfrak{B}^{\prime} \text { are disjoint and both cross the annulus }\right\} .
\end{aligned}
$$

When $\mathcal{E}$ holds, let $\mathfrak{L}_{B}$ be the $\pi$-extremal distance between $C_{r}$ and $C$ in $A(r, 1) \backslash \mathfrak{B}$. Similarly, when $\tilde{\mathcal{E}}$ is satisfied, let $O_{1}$ and $O_{2}$ be the two components of $A(r, 1) \backslash\left(\mathfrak{B} \cup \mathfrak{B}^{\prime}\right)$ which have arcs of $C$ on their boundaries, in such a way that the sequence $\mathfrak{B}, O_{1}, \mathfrak{B}^{\prime}, O_{2}$ is in counterclockwise order around $A(r, 1)$. Let $\mathfrak{L}_{1}$ be the $\pi$-extremal distance from $C_{r}$ to $C$ in $O_{1}$, and let $\mathfrak{L}_{2}$ be the corresponding quantity for $O_{2}$. Then for $\lambda, \lambda_{1}, \lambda_{2} \in \mathbf{R}_{+}$, the exponents $\xi(1, \lambda)$ and $\xi\left(1, \lambda_{1}, 1, \lambda_{2}\right)$ can be described [32] as

$$
\int_{\mathcal{E}} \exp (-\lambda \mathfrak{L}) d \mu_{r}^{1}(B) \approx r^{\xi(1, \lambda)}
$$

and

$$
\int_{\tilde{\mathcal{E}}} \exp \left(-\lambda_{1} \mathfrak{L}_{1}-\lambda_{2} \mathfrak{L}_{2}\right) d \mu_{r}^{1}(B) d \mu_{r}^{1}\left(B^{\prime}\right) \approx r^{\xi\left(1, \lambda_{1}, 1, \lambda_{2}\right)}
$$

as $r \searrow 0$.

\subsection{A useful technical lemma}

We now derive a technical refinement of (5.18) and (5.19) (in the case $\lambda_{1}=1$ ) that will be useful to identify the Brownian intersection exponents with those computed for $\mathrm{SLE}_{6}$.

Keep the same notation as above, and on $\mathcal{E}$, let $\phi$ denote the conformal map that maps $O$ onto $R_{\mathfrak{L}}$ in such a way that the images of $C_{1} \cap \bar{O}$ and $C_{r} \cap \bar{O}$ are mapped onto the left and right edges of the rectangle, respectively. Similarly, on $\tilde{\mathcal{E}}$, let $\phi_{1}$ be the corresponding conformal map from $O_{1}$ onto $R_{\mathfrak{L}_{1}}$. For all $\alpha>0$ set

$$
\begin{aligned}
& \mathcal{H}_{\alpha}:=\mathcal{E} \cap\{i \in \bar{O} \text { and } \phi(i) \in[i \alpha, i(\pi-\alpha)]\}, \\
& \widetilde{\mathcal{H}}_{\alpha}:=\tilde{\mathcal{E}} \cap\left\{i \in \bar{O}_{1} \text { and } \phi_{1}(i) \in[i \alpha, i(\pi-\alpha)]\right\}
\end{aligned}
$$

LEMmA 5.5. Let $\lambda>0$. Then there are sequences $x_{n} \searrow^{0}$ and $y_{n} \searrow 0$, and an $\alpha>0$, such that

$$
\begin{gathered}
\int_{\mathcal{H}_{\alpha}} \exp \left(-\lambda \mathfrak{L}_{B}\right) d \mu_{x_{n}}^{1}(B) \approx\left(x_{n}\right)^{\xi(1, \lambda)}, \quad n \rightarrow \infty, \\
\int_{\widetilde{\mathcal{H}}_{\alpha}} \exp \left(-\mathfrak{L}_{1}-\lambda \mathfrak{L}_{2}\right) d \mu_{y_{n}}^{1}(B) d \mu_{y_{n}}^{1}\left(B^{\prime}\right) \approx\left(y_{n}\right)^{\xi(1,1,1, \lambda)}, \quad n \rightarrow \infty .
\end{gathered}
$$


Actually (see, e.g., [30]), much stronger statements hold: In the above, $\approx$ may be replaced by $\asymp$, and these statements hold for every sequence tending to $\infty$. But the present statement will be sufficient to determine the values of the Brownian intersection exponents, and it can be easily proved as follows.

Proof. We will only give the detailed proof of (5.21). The proof of (5.20) is easier, follows exactly the same lines, and is safely left to the reader.

Because of (5.19), it suffices to find the lower bound for the left-hand side of (5.21). Let us first introduce some notation. Let $r \in\left(0, \frac{1}{4}\right)$, and consider the measure $\mu_{r}^{1} \times \mu_{r}^{1}$ on the space of pairs $\left(B, B^{\prime}\right)$. Let

$$
f(r):=\int_{\overline{\mathcal{E}}(r)} \exp \left(-\mathfrak{L}_{1}-\lambda \mathfrak{L}_{2}\right) d \mu_{r}^{1}(B) d \mu_{r}^{1}\left(B^{\prime}\right) .
$$

Let $B^{*}$ be the path $B$ stopped when it hits $C_{1 / 4}$, if it does, and $B^{*}=B$, if it does not hit $C_{1 / 4}$. Similarly, define $B^{\prime *}$ from $B^{\prime}$. Let $\mathfrak{B}$ and $\mathfrak{B}^{\prime}$ be the traces of $B$ and $B^{\prime}$, respectively. Let $\tilde{\mathcal{E}}^{*}$ be the event that the traces of $B^{*}$ and $B^{\prime *}$ are disjoint. On $\tilde{\mathcal{E}}^{*}$, let $O_{1}^{*}$ and $O_{2}^{*}$ be the domains defined for $B^{*}$ and $B^{\prime *}$, as $O_{1}$ and $O_{2}$ were defined for $B$ and $B^{\prime}$. Then $O_{j}^{*} \subset O_{j}$ on $\tilde{\mathcal{E}}, j=1,2$. For $j=1,2$, on $\tilde{\mathcal{E}}^{*}$, let $\mathfrak{L}_{j}^{*}$ be the $\pi$-extremal distance between $C_{r}$ and $C_{1 / 4}$ in $O_{j}^{*}$. Otherwise, set $\mathfrak{L}_{j}^{*}=\infty$.

For $a>0$, let $\mathcal{D}_{a}$ be the event that the distance between $\mathfrak{B} \cap A\left(\frac{1}{2}, 1\right)$ and $\mathfrak{B}^{\prime} \cap A\left(\frac{1}{2}, 1\right)$ is at least $a$. Suppose that $a \in\left(0, \frac{1}{5}\right)$. Observe that for $\left(B, B^{\prime}\right) \notin \mathcal{D}_{a}$, there is for $j=1$ or $j=2$ a path of length at most $a$ in $O_{j} \cap A\left(\frac{1}{2}, 1\right)$ which separates $C_{r}$ from $C_{1}$ in $O_{j}$. It then follows that $\mathfrak{L}_{j} \geqslant \mathfrak{L}_{j}^{*}+c_{1} \log (1 / a)$, for some constant $c_{1}>0$. Consequently,

$$
\begin{aligned}
\int_{\tilde{\mathcal{E}}(r) \backslash \mathcal{D}_{a}} \exp (- & \left.\mathfrak{L}_{1}-\lambda \mathfrak{L}_{2}\right) d \mu_{r}^{1}(B) d \mu_{r}^{1}\left(B^{\prime}\right) \\
& \leqslant a^{c_{1} \min \{\lambda, 1\}} \int_{\tilde{\mathcal{E}}^{*}} \exp \left(-\mathfrak{L}_{1}^{*}-\lambda \mathfrak{L}_{2}^{*}\right) d \mu_{r}^{1}(B) d \mu_{r}^{1}\left(B^{\prime}\right) \\
& =a^{c_{1} \min \{\lambda, 1\}} f(4 r),
\end{aligned}
$$

since the image of $\mu_{r}^{1}$ under the map $B \mapsto 4 B^{*}$ is $\mu_{4 r}^{1}$.

By (5.19), we have $f(r) \approx r^{\xi(1,1, \lambda, 1)}$ (and $f$ is non-decreasing). Consequently, if $a$ is chosen sufficiently small, there is a sequence $y_{n} \searrow 0$ (for instance a subsequence of $4^{-n}$ ) such that $f\left(y_{n}\right) \geqslant 2 a^{c_{1} \min \{\lambda, 1\}} f\left(4 y_{n}\right)$. For these $y_{n},(5.22)$ gives

$$
\int_{\tilde{\mathcal{E}}\left(y_{n}\right) \cap \mathcal{D}_{a}} \exp \left(-\mathfrak{L}_{1}-\lambda \mathfrak{L}_{2}\right) d \mu_{y_{n}}^{1}(B) d \mu_{y_{n}}^{1}\left(B^{\prime}\right) \geqslant a^{c_{1} \min \{\lambda, 1\}} f\left(4 y_{n}\right) .
$$

Fix such an $a$ and such a $y_{n}$. Let $\mathcal{I}_{a}$ be the event that $i \in \bar{O}_{1}$ and the distance from $i$ to $\mathfrak{B} \cup \mathfrak{B}^{\prime}$ is at least $\frac{1}{10} a$. Observe that if we apply an independent random uniform rotation 
about 0 to a pair $\left(B, B^{\prime}\right) \in \mathcal{D}_{a} \cap \tilde{\mathcal{E}}$, then with probability at least $a / 10 \pi$ the rotated pair is in $\mathcal{I}_{a}$. Since the integrand in (5.23) is invariant under rotations, (5.23) and (5.19) give

$$
\int_{\tilde{\mathcal{E}}\left(y_{n}\right) \cap \mathcal{I}_{a}} \exp \left(-\mathfrak{L}_{1}-\lambda \mathfrak{L}_{2}\right) d \mu_{y_{n}}^{1}(B) d \mu_{y_{n}}^{1}\left(B^{\prime}\right) \approx\left(y_{n}\right)^{\xi(1,1, \lambda, 1)}
$$

It therefore suffices to show that when $\alpha>0$ is small, we have $\mathcal{I}_{a} \cap \tilde{\mathcal{E}} \subset \widetilde{\mathcal{H}}_{\alpha}$. To prove this, consider a pair $\left(B, B^{\prime}\right) \in \mathcal{I}_{a}$. Let $A$ be the subarc of $\bar{O}_{1} \cap C_{1}$ that has $i$ as one endpoint and the other endpoint is in $\mathfrak{B}$, and let $A^{\prime}$ be the subarc of $\bar{O}_{1} \cap C_{1}$ that has $i$ as one endpoint and the other endpoint is in $\mathfrak{B}^{\prime}$. Then the extremal distance from $A$ to $\mathfrak{B}^{\prime}$ in $O_{1}$ is bounded from below by a positive constant depending only on $a$, as is the extremal distance from $A^{\prime}$ to $\mathfrak{B}$ in $O_{1}$. Conformal invariance of extremal distance therefore shows that the distance from $\phi(i)$ to $\{0, i \pi\}$ is bounded from below by a constant depending only on $a$, which proves that $\left(B, B^{\prime}\right) \in \widetilde{\mathcal{H}}_{\alpha}$, where $\alpha>0$ depends only on $a$.

\section{The universality argument}

We are now ready to combine the results derived so far to prove our main theorems. As in [27], we follow the universality ideas presented in [32]. First, Theorem $5.6\left(\xi(1,1)=\frac{5}{4}\right)$ will be proved, followed by Theorem 5.7 (giving $\xi(1,1,1, \lambda)$ ). As we have seen, Theorems 1.1 and 1.2 are immediate consequences.

\subsection{Proof of $\xi(1,1)=\frac{5}{4}$}

Let $r>0$, let $K_{t}$ be a radial $\mathrm{SLE}_{6}$-process in $\mathbf{U}$ starting at $i$, and let $T=T(r)$ be the least $t$ such that $\bar{K}_{t} \cap C_{r} \neq \varnothing$. Set $\mathfrak{K}:=\bar{K}_{T}$, let $\mathbf{P}_{r}$ denote the law of $\mathfrak{K}$, and let $\mathbf{E}_{r}$ denote expectation with respect to this measure. As before, we let $\mu_{r}^{1}$ denote the Brownian excursion measure in $A(r, 1)$ started from $C_{r}$, and let $\mathfrak{B}$ denote the trace of the excursion $B$. We are interested in the event $\mathcal{E}^{*}$ in which $\mathfrak{K} \cap \mathfrak{B}=\varnothing$ and $\mathfrak{B}$ crosses $A(r, 1)$ (i.e., $\mathfrak{B} \cap C_{1} \neq \varnothing$ ).

The proof will proceed by computing $\left(\mathbf{P}_{r} \times \mu_{r}^{1}\right)\left[\mathcal{E}^{*}\right]$ in two different ways. In the first computation, we begin by conditioning on $\mathfrak{K}$ and then taking the expectation, while the second computation begins by conditioning on $B$.

When $\mathfrak{B}$ does not separate $C_{r}$ from $C$ in $\mathbf{U}$, let $O_{B}$ denote the connected component of $A(r, 1) \backslash \mathfrak{B}$ that touches both circles $C_{r}$ and $C$, and $\mathfrak{L}_{B}$ the corresponding $\pi$-extremal distance.

When $\mathfrak{K}$ does not disconnect $C_{r}$ from $C$ in $\mathbf{U}$, let $O_{\mathfrak{K}}$ denote the connected component of $A(r, 1) \backslash \mathfrak{K}$ that touches both circles, and $\mathfrak{L}_{\mathfrak{K}}$ the corresponding $\pi$-extremal distance. 
Suppose first that $\mathfrak{K}$ is given and that $\mathfrak{L}_{\mathfrak{K}}<\infty$. Note that $\mathfrak{L}_{\mathfrak{K}} \rightarrow \infty$ as $r \searrow 0$. By the restriction property and conformal invariance of the Brownian excursion measure (see $§ 5.2$ ) and Lemma 5.4,

$$
\mu_{r}^{1}\left[B \text { crosses the annulus in } O_{\mathfrak{R}}\right]=\mu_{R_{\mathfrak{L}_{\mathfrak{K}}}}\left[\mathcal{E}_{\mathfrak{L}_{\mathfrak{K}}}\right] \asymp \exp \left(-\mathfrak{L}_{\mathfrak{K}}\right), \quad r \searrow 0,
$$

where $\mathcal{E}_{L}$ is as in Lemma 5.4. On the other hand, we know from Theorem 3.1, with $b=1$, $\kappa=6$, that

$$
\mathbf{E}_{r}\left[\exp \left(-\mathfrak{L}_{\mathfrak{K}}\right)\right] \approx r^{5 / 4}, \quad r \searrow 0
$$

Combining these two facts implies that

$$
\left(\mathbf{P}_{r} \times \mu_{r}^{1}\right)\left[\mathcal{E}^{*}\right] \approx r^{5 / 4}, \quad r \searrow 0
$$

Suppose now that $B$ is given and that it does cross the annulus without separating the disk $C_{r}$ from $C$. The second part of Lemma 4.3 shows that there exists a constant $c>0$ such that

$$
\mathbf{P}_{r}\left[\mathcal{E}^{*}\right] \leqslant c \exp \left(-\mathfrak{L}_{B}\right)
$$

Hence, combining this with (5.18) shows that

$$
\left(\mathbf{P}_{r} \times \mu_{r}^{1}\right)\left[\mathcal{E}^{*}\right] \leqslant r^{\xi(1,1)+o(1)}
$$

On the other hand, suppose now that $B$ is given and that $B \in \mathcal{H}_{\alpha}$, where $\mathcal{H}_{\alpha}$ is as in Lemma 5.5. Then by the first part of Lemma 4.3, there exists a constant $c^{\prime}$ such that

$$
\mathbf{P}_{r}\left[\mathcal{E}^{*}\right] \geqslant c^{\prime} \exp \left(-\mathfrak{L}_{B}\right)
$$

Combining this with Lemma 5.5, we find that

$$
\left(\mathbf{P}_{x_{n}} \times \mu_{x_{n}}^{1}\right)\left[\mathcal{E}^{*}\right] \geqslant\left(\mathbf{P}_{x_{n}} \times \mu_{x_{n}}^{1}\right)\left[\mathcal{H}_{\alpha} \cap \mathcal{E}^{*}\right] \geqslant\left(x_{n}\right)^{\xi(1,1)+o(1)}, \quad n \rightarrow \infty
$$

Comparing with (6.2) gives

$$
\left(\mathbf{P}_{x_{n}} \times \mu_{x_{n}}^{1}\right)\left[\mathcal{E}^{*}\right] \approx\left(x_{n}\right)^{\xi(1,1)}, \quad n \rightarrow \infty
$$

Now, by (6.1),

$$
\left(x_{n}\right)^{5 / 4} \approx\left(\mathbf{P}_{x_{n}} \times \mu_{x_{n}}^{1}\right)\left[\mathcal{E}^{*}\right] \approx\left(x_{n}\right)^{\xi(1,1)}
$$

when $n \rightarrow \infty$, which proves $\xi(1,1)=\frac{5}{4}$. 


\subsection{The determination of $\xi(1,1,1, \lambda)$}

The goal now is to prove (5.7). As $\lambda \mapsto \xi(1,1,1, \lambda)=\xi(1,1, \tilde{\xi}(1, \lambda))$ is continuous on $[0, \infty)$ (see [31]), we can restrict ourselves to the case where $\lambda>0$. The proof goes along similar lines as the proof of $\xi(1,1)=\frac{5}{4}$. This time, we will consider two independent Brownian excursions $B$ and $B^{\prime}$ in the annulus $A(r, 1)$, and one $\mathrm{SLE}_{6} \mathfrak{K}$ as before. We are interested in the event that $B, B^{\prime}$ and $\mathfrak{K}$ all cross the annulus, that they remain disjoint, and that $B, \mathfrak{K}$ and $B^{\prime}$ are in clockwise order. Let us call this event $l$. Note that in this case, $\mathfrak{K}$ crosses the annulus in $O_{1}$, where we use the notations of $\S 5.3$.

We shall compute in two different ways the quantity

$$
\int_{l} \exp \left(-\lambda \mathfrak{L}_{2}\right) d \mu_{r}^{1}(B) d \mu_{r}^{1}\left(B^{\prime}\right) d \mathbf{P}_{r}(\mathfrak{K}) .
$$

On the one hand, we know from conformal invariance and the restriction property of Brownian excursions and from (5.17) that when $\mathfrak{K}$ crosses the annulus without separating $C_{r}$ from $C$,

$$
\int_{l} \exp \left(-\lambda \mathfrak{L}_{2}\right) d \mu_{r}^{1}(B) d \mu_{r}^{1}\left(B^{\prime}\right) \approx \exp \left(-\mathfrak{L}_{\mathfrak{K}} \tilde{\xi}(1,1, \lambda)\right), \quad r \searrow 0 .
$$

But we know from Theorem 3.1 that

$$
\int \exp \left(-b \mathfrak{L}_{\mathfrak{K}}\right) d \mathbf{P}_{r} \approx r^{\nu}
$$

where

$$
\nu=\nu(b)=\frac{1}{8}(4 b+1+\sqrt{1+24 b}) .
$$

Note that $\nu$ is a continuous increasing function of $b$ on $(0, \infty)$. Consequently, $(6.3)$ and (6.4) give

$$
\int_{l} \exp \left(-\lambda \mathfrak{L}_{2}\right) d \mu_{r}^{1}(B) d \mu_{r}^{1}\left(B^{\prime}\right) d \mathbf{P}_{r}(\mathfrak{K}) \approx r^{\nu(\tilde{\xi}(1,1, \lambda))} .
$$

Combining this with (5.4) shows that

$$
\int_{l} \exp \left(-\lambda \mathfrak{L}_{2}\right) d \mu_{r}^{1}(B) d \mu_{r}^{1}\left(B^{\prime}\right) d \mathbf{P}_{r}(\mathfrak{K}) \approx r^{a},
$$

where

$$
a=a(\lambda):=\nu(\tilde{\xi}(1,1, \lambda))=\frac{1}{48}\left((11+\sqrt{24 \lambda+1})^{2}-4\right) .
$$

Suppose now that $B$ and $B^{\prime}$ are given and that $\tilde{\mathcal{E}}$ is satisfied; i.e., that $B$ and $B^{\prime}$ cross the annulus without intersecting each other. Then Lemma 4.3 shows that

$$
\mathbf{P}_{r}\left[\mathfrak{K} \subset O_{1}\right] \leqslant c \exp \left(-\mathfrak{L}_{1}\right)
$$


for some constant $c$. Hence, combining this with (5.19) shows that

$$
\int_{l} \exp \left(-\lambda \mathfrak{L}_{2}\right) d \mathbf{P}_{r}(\mathfrak{K}) d \mu_{r}^{1}(B) d \mu_{r}^{1}\left(B^{\prime}\right) \leqslant r^{\xi(1,1,1, \lambda)+o(1)} .
$$

Suppose now that $B$ and $B^{\prime}$ are given and that $\left(B, B^{\prime}\right) \in \widetilde{\mathcal{H}}_{\alpha}$. Then Lemma 4.3 shows that there exists a constant $c^{\prime}>0$ such that

$$
\mathbf{P}_{r}\left[\mathfrak{K} \subset O_{1}\right] \geqslant c^{\prime} \exp \left(-\mathfrak{L}_{1}\right)
$$

Combining this with Lemma 5.5 gives

$$
\int_{l} \exp \left(-\lambda \mathfrak{L}_{2}\right) d \mathbf{P}_{y_{n}}(\mathfrak{K}) d \mu_{y_{n}}^{1}(B) d \mu_{y_{n}}^{1}\left(B^{\prime}\right) \geqslant\left(y_{n}\right)^{\xi(1,1,1, \lambda)+o(1)} .
$$

Comparing with (6.6) implies that

$$
\int_{l} \exp \left(-\lambda \mathfrak{L}_{2}\right) d \mathbf{P}_{y_{n}}(\mathfrak{K}) d \mu_{y_{n}}^{1}(B) d \mu_{y_{n}}^{1}\left(B^{\prime}\right) \approx\left(y_{n}\right)^{\xi(1,1,1, \lambda)},
$$

when $n \rightarrow \infty$. Consequently, by $(6.5), \xi(1,1,1, \lambda)=a(\lambda)$. 


\section{References}

[1] Ahlfors, L. V., Conformal Invariants: Topics in Geometric Function Theory. McGrawHill, New York, 1973.

[2] Aizenman, M., Duplantier, B. \& Aharony, A., Path crossing exponents and the external perimeter in 2D percolation. Phys. Rev. Lett., 83 (1999), 1359-1362.

[3] Azencott, R., Behaviour of diffusion semi-groups at infinity. Bull. Soc. Math. France, 102 (1974), 193-240.

[4] Bishop, C. J., Jones, P. W., Pemantle, R. \& Peres, Y., The dimension of the Brownian frontier is greater than 1. J. Funct. Anal., 143 (1997), 309-336.

[5] Burdzy, K. \& Lawler, G.F., Non-intersection exponents for Brownian paths. Part I: Existence and an invariance principle. Probab. Theory Related Fields, 84 (1990), 393-410.

[6] - Non-intersection exponents for Brownian paths. Part II: Estimates and applications to a random fractal. Ann. Probab., 18 (1990), 981-1009.

[7] Cardy, J. L., Conformal invariance and surface critical behavior. Nuclear Phys. B, 240 (1984), 514-532.

[8] - Critical percolation in finite geometries. J. Phys. A, 25 (1992), L201-L206.

[9] - The number of incipient spanning clusters in two-dimensional percolation. J. Phys. A, 31 (1998), L105.

[10] Cranston, M. \& Mountford, T., An extension of a result of Burdzy and Lawler. Probab. Theory Related Fields, 89 (1991), 487-502.

[11] Duplantier, B., Random walks and quantum gravity in two dimensions. Phys. Rev. Lett., 81 (1998), 5489-5492.

[12] - Two-dimensional copolymers and exact conformal multifractality. Phys. Rev. Lett., 82 (1999), 880-883.

[13] - Harmonic measure exponents for two-dimensional percolation. Phys. Rev. Lett., 82 (1999), 3940-3943.

[14] Duplantier, B. \& KWon, K.-H., Conformal invariance and intersection of random walks. Phys. Rev. Lett., 61 (1988), 2514-2517.

[15] Duplantier, B. \& Saleur, H., Exact determination of the percolation hull exponent in two dimensions. Phys. Rev. Lett., 58 (1987), 2325-2328.

[16] Kenyon, R., Conformal invariance of domino tiling. Ann. Probab., 28 (2000), 759-795.

[17] - Long-range properties of spanning trees. Probabilistic techniques in equilibrium and nonequilibrium statistical physics. J. Math. Phys., 41 (2000), 1338-1363.

[18] - The asymptotic determinant of the discrete Laplacian. Acta Math., 185 (2000), 239-286.

[19] LAWLER, G. F., Intersections of Random Walks. Birkhäuser Boston, Boston, MA, 1991.

[20] - Hausdorff dimension of cut points for Brownian motion. Electron. J. Probab., 1:2 (1996), 1-20 (electronic).

[21] - Cut times for simple random walk. Electron. J. Probab., 1:13 (1996), 1-24 (electronic).

[22] - The dimension of the frontier of planar Brownian motion. Electron. Comm. Probab., 1:5 (1996), 29-47 (electronic).

[23] - The frontier of a Brownian path is multifractal. Preprint, 1997.

[24] - Strict concavity of the intersection exponent for Brownian motion in two and three dimensions. Math. Phys. Electron. J., 4:5 (1998), 1-67 (electronic).

[25] - Geometric and fractal properties of Brownian motion and random walk paths in two and three dimensions, in Random Walks (Budapest, 1998), pp. 219-258. Bolyai Soc. Math. Stud., 9. János Bolyai Math. Soc., Budapest, 1999.

[26] Lawler, G.F. \& Puckette, E. E., The intersection exponent for simple random walk. Combin. Probab. Comput., 9 (2000), 441-464. 
[27] Lawler, G.F., Schramm, O. \& Werner, W., Values of Brownian intersection exponents, I: Half-plane exponents. Acta Math., 187 (2001), 237-273.

[28] - Values of Brownian intersection exponents, III: Two-sided exponents. To appear in Ann. Inst. H. Poincaré Probab. Statist. http://arxiv.org/abs/math.PR/0005294.

[29] - Analyticity of intersection exponents for planar Brownian motion. To appear in Acta Math., 188 (2002). http://arxiv.org/abs/math.PR/0005295.

[30] - Sharp estimates for Brownian non-intersection probabilities. To appear in In and Out of Equilibrium. Probability with a Physics Flavor. Progr. Probab. Birkhäuser Boston, Boston, MA.

[31] LaWler, G. F. \& Werner, W., Intersection exponents for planar Brownian motion. Ann. Probab., 27 (1999), 1601-1642.

[32] - Universality for conformally invariant intersection exponents. J. Eur. Math. Soc. (JEMS), 2 (2000), 291-328.

[33] LöWNER, K., Untersuchungen über schlichte konforme Abbildungen des Einheitskreises, I. Math. Ann., 89 (1923), 103-121.

[34] Madras, N. \& Slade, G., The Self-Avoiding Walk. Birkhäuser Boston, Boston, MA, 1993.

[35] Mandelbrot, B. B., The Fractal Geometry of Nature. Freeman, San Francisco, CA, 1982.

[36] NiEnhuis, B., Critical behavior of two-dimensional spin models and charge asymmetry in the Coulomb gas. J. Statist. Phys., 34 (1984), 731-761.

[37] Pommerenke, Ch., On the Loewner differential equation. Michigan Math. J., 13 (1966), 435-443.

[38] Revuz, D. \& Yor, M., Continuous Martingales and Brownian Motion. Grundlehren Math. Wiss., 293. Springer-Verlag, Berlin, 1991.

[39] Schramm, O., Scaling limits of loop-erased random walks and uniform spanning trees. Israel J. Math., 118 (2000), 221-288.

[40] Werner, W., Bounds for disconnection exponents. Electron. Comm. Probab., 1:4 (1996), 19-28 (electronic).

Gregory F. LAWLer

Department of Mathematics

Duke University

Box 90320

Durham, NC 27708-0320

U.S.A.

jose@math.duke.edu

WENDELIN WERNER

Département de Mathématiques

Université Paris-Sud

Bât. 425

FR-91405 Orsay Cedex

France

wendelin.werner@math.u-psud.fr

Received May 18, 2000
Oded Schramm

Microsoft Research

1, Microsoft Way

Redmond, WA 98052

U.S.A.

schramm@microsoft.com 